\title{
McCool groups of toral relatively hyperbolic groups
}

\author{
VINCENT GUIRARDEL \\ GILBERT LEVITT
}

\begin{abstract}
The outer automorphism group $\operatorname{Out}(G)$ of a group $G$ acts on the set of conjugacy classes of elements of $G$. McCool proved that the stabilizer $\operatorname{Mc}(\mathcal{C})$ of a finite set of conjugacy classes is finitely presented when $G$ is free. More generally, we consider the group $\operatorname{Mc}(\mathcal{H})$ of outer automorphisms $\Phi$ of $G$ acting trivially on a family of subgroups $H_{i}$, in the sense that $\Phi$ has representatives $\alpha_{i}$ that are equal to the identity on $H_{i}$.
\end{abstract}

When $G$ is a toral relatively hyperbolic group, we show that these two definitions lead to the same subgroups of $\operatorname{Out}(G)$, which we call "McCool groups" of G. We prove that such McCool groups are of type VF (some finite-index subgroup has a finite classifying space). Being of type VF also holds for the group of automorphisms of $G$ preserving a splitting of $G$ over abelian groups.

We show that McCool groups satisfy a uniform chain condition: there is a bound, depending only on $G$, for the length of a strictly decreasing sequence of McCool groups of $G$. Similarly, fixed subgroups of automorphisms of $G$ satisfy a uniform chain condition.

20F28; 20F65, 20F67

\section{Introduction}

Mapping class groups of punctured surfaces may be viewed as subgroups of $\operatorname{Out}\left(F_{n}\right)$ for some $n$ (with $F_{n}$ denoting the free group of rank $n$ ). Indeed, they consist of automorphisms of $F_{n}$ fixing conjugacy classes corresponding to punctures. More generally, the group of automorphisms of $F_{n}$ fixing a finite number of conjugacy classes was studied by McCool [30], who proved in particular that such groups are finitely presented. We therefore define:

Definition 1.1 Let $G$ be a group. Let $\mathcal{C}$ be a set of conjugacy classes $\left[c_{i}\right]$ of elements of $G$. We denote by $\operatorname{Mc}(\mathcal{C})$ the subgroup of $\operatorname{Out}(G)$ consisting of outer automorphisms fixing each $\left[c_{i}\right]$. If $\mathcal{C}$ is finite, we say that $\operatorname{Mc}(\mathcal{C})$ is an elementary McCool group of $G$ (or of $\operatorname{Out}(G)$ ). 
Work on automorphisms suggests a more general definition:

Definition 1.2 Let $G$ be a group. Let $\mathcal{H}=\left\{H_{i}\right\}$ be an arbitrary family of subgroups of $G$. We say that $\varphi \in \operatorname{Aut}(G)$ and its image $\Phi \in \operatorname{Out}(G)$ act trivially on $\mathcal{H}$ if $\varphi$ acts on each $H_{i}$ as conjugation by some $g_{i} \in G$. Note that $\Phi$ acts trivially if and only if it has representatives $\varphi_{i} \in \operatorname{Aut}(G)$ with $\varphi_{i}$ equal to the identity on $H_{i}$.

We denote by $\operatorname{Mc}(\mathcal{H})$ or $\operatorname{Mc}_{G}(\mathcal{H})$ the subgroup of $\operatorname{Out}(G)$ consisting of all $\Phi$ acting trivially on $\mathcal{H}$.

If $\mathcal{H}$ is a finite family of finitely generated subgroups, we say that $\operatorname{Mc}(\mathcal{H})$ is a $\mathrm{McCool}$ group of $G$ (or of $\operatorname{Out}(G))$.

Elementary $\mathrm{McCool}$ groups correspond to $\mathrm{McCool}$ groups with $\mathcal{H}$ a finite family of cyclic groups. $\operatorname{Mc}(\mathcal{H})$ does not change if we replace the $H_{i}$ by conjugate subgroups, so it is really associated to a family of conjugacy classes of subgroups.

For a topological analogy, one may think of $\operatorname{Mc}(\mathcal{H})$ as the group of automorphisms of $G=\pi_{1}(X)$ induced by homeomorphisms of $X$ equal to the identity on subspaces $Y_{i}$ with $\pi_{1}\left(Y_{i}\right)=H_{i}$.

McCool groups are relevant for automorphisms for the following reason (see Guirardel and Levitt [25]). Consider a splitting of a group $\widehat{G}$ as a graph of groups in which $G$ is a vertex group and the $H_{i}$ are the incident edge groups. Then any element of $\operatorname{Mc}_{G}(\mathcal{H})$ extends "by the identity" to an automorphism of $\widehat{G}$. Topologically, if $X$ is a vertex space in a graph of spaces $\hat{X}$ and edge spaces are attached to subspaces $Y_{i} \subset X$, then any homeomorphism of $X$ equal to the identity on the $Y_{i}$ extends to $\widehat{X}$ by the identity.

In this paper we will consider $\mathrm{McCool}$ groups when $G$ is a toral relatively hyperbolic group: $G$ is torsion-free and hyperbolic relative to a finite set of finitely generated abelian subgroups. This includes in particular torsion-free hyperbolic groups, limit groups and groups acting freely on $\mathbb{R}^{n}$-trees.

We will show (Corollary 1.6) that in this case any $\operatorname{Mc}(\mathcal{H})$ is an elementary McCool group $\operatorname{Mc}(\mathcal{C})$; in other words, it is equivalent for a subgroup of $\operatorname{Out}(G)$ to be an elementary $\mathrm{McCool}$ group $\operatorname{Mc}(\mathcal{C})$, or to be a $\operatorname{McCool}$ group $\operatorname{Mc}(\mathcal{H})$ with $\mathcal{H}$ a finite family of finitely generated groups, or to be $\operatorname{Mc}(\mathcal{H})$ with $\mathcal{H}$ arbitrary. We will not always make the distinction in the statements given below.

It was proved by $\mathrm{McCool}$ [30] that (elementary) $\mathrm{McCool}$ groups of a free group are finitely presented. Culler and Vogtmann [9, Corollary 6.1.4] proved that they are of type VF: they have a finite-index subgroup with a finite classifying space (ie there exists a classifying space which is a finite complex). We proved in [25] that $\operatorname{Out}(G)$ 
is of type VF if $G$ is toral relatively hyperbolic (in particular, $\operatorname{Out}(G)$ is virtually torsion-free). Our first main results extend this to certain naturally defined subgroups of $\operatorname{Out}(G)$.

Theorem 1.3 If $G$ is a toral relatively hyperbolic group, then any McCool group $\operatorname{Mc}(\mathcal{H}) \subset \operatorname{Out}(G)$ is of type VF.

Theorem 1.4 If $G$ is a toral relatively hyperbolic group and $T$ is a simplicial tree on which $G$ acts with abelian edge stabilizers, then the group of automorphisms $\operatorname{Out}(T) \subset \operatorname{Out}(G)$ leaving $T$ invariant is of type VF.

Our most general result in this direction (Corollary 6.3) combines these two theorems; it implies in particular that $\operatorname{Mc}(\mathcal{H}) \cap \operatorname{Out}(T)$ is of type $\mathrm{VF}$ if $T$ is as above and $\mathcal{H}$ is any family of subgroups each of which fixes a point in $T$.

Remark Some of these results may be extended to groups which are hyperbolic relative to virtually polycyclic subgroups, but with the weaker conclusion that the automorphism groups are of type $\mathrm{F}_{\infty}$ (see Guirardel and Levitt [17]). On the other hand, one can show that, if there exists a hyperbolic group which is not residually finite, then there exists a hyperbolic group with $\operatorname{Out}(G)$ not virtually torsion-free (hence not VF).

Our second main result is the following:

Theorem 1.5 Let $G$ be a toral relatively hyperbolic group. McCool groups of $G$ satisfy a uniform chain condition: there exists $C=C(G)$ such that, if

$$
\operatorname{Mc}\left(\mathcal{H}_{0}\right) \supsetneq \operatorname{Mc}\left(\mathcal{H}_{1}\right) \supsetneq \cdots \supsetneq \operatorname{Mc}\left(\mathcal{H}_{p}\right)
$$

is a strictly decreasing chain of $\mathrm{McCool}$ groups in $\operatorname{Out}(G)$, then $p \leq C$.

This is based, among other things, on the vertex finiteness we proved in [24]: if $G$ is toral relatively hyperbolic, then all vertex groups occurring in splittings of $G$ over abelian groups lie in finitely many isomorphism classes.

The chain condition, proved in Section 5 for $\mathrm{McCool}$ groups $\operatorname{Mc}(\mathcal{H})$ with $\mathcal{H}$ a finite family of finitely generated groups, implies:

Corollary 1.6 Let $G$ be a toral relatively hyperbolic group. If $\mathcal{H}$ is a (possibly infinite) family of (possibly infinitely generated) subgroups $H_{i} \subset G$, there exists a finite set of conjugacy classes $\mathcal{C}$ such that $\operatorname{Mc}(\mathcal{H})=\operatorname{Mc}(\mathcal{C})$. In particular, any $\operatorname{Mc}(\mathcal{H})$ is a McCool group and any $\mathrm{McCool}$ group is an elementary $\mathrm{McCool}$ group $\mathrm{Mc}(\mathcal{C})$. 
The chain condition also implies that no $\operatorname{McCool} \operatorname{group} \operatorname{Mc}(\mathcal{H}) \subset \operatorname{Out}(G)$ is conjugate to a proper subgroup. Note, however, that $\mathrm{McCool}$ groups may fail to be co-Hopfian (they may be isomorphic to proper subgroups). To illustrate the variety of McCool groups, we show:

Proposition $1.7 \operatorname{Out}\left(F_{n}\right)$ contains infinitely many non-isomorphic McCool groups if $n \geq 4$; it contains infinitely many non-conjugate McCool groups if $n \geq 3$.

It may be shown that the bounds on $n$ are sharp (see the appendix). We will also show in the appendix that, if $G$ is a torsion-free, one-ended hyperbolic group, then $\operatorname{Out}(G)$ only contains finitely many McCool groups up to conjugacy.

Say that $J \subset G$ is a fixed subgroup if there is a family of automorphisms $\alpha_{i} \in \operatorname{Aut}(G)$ such that $J=\bigcap_{i}$ Fix $\alpha_{i}$, with Fix $\alpha=\{g \in G \mid \alpha(g)=g\}$. The chain condition also implies:

Theorem 1.8 Let $G$ be a toral relatively hyperbolic group. There is a constant $c=c(G)$ such that, if $J_{0} \varsubsetneqq J_{1} \varsubsetneqq \cdots \varsubsetneqq J_{p}$ is a strictly ascending chain of fixed subgroups, then $p \leq c$.

This was proved by Martino and Ventura [29] for $G$ free, with $c\left(F_{n}\right)=2 n$. In [18], we will apply Theorems 1.3 and 1.8 to the study of stabilizers for the action of $\operatorname{Out}(G)$ on spaces of $\mathbb{R}$-trees.

As explained above, one does not get new groups by allowing the set $\mathcal{C}$ in Definition 1.1 to be infinite or by considering arbitrary subgroups as in Definition 1.2. The following definition provides a genuine generalization.

Definition 1.9 Let $G$ be a group, and $\mathcal{C}$ a finite set of conjugacy classes $\left[c_{i}\right]$. We write $\mathcal{C}^{-1}$ for the set of classes $\left[c_{i}^{-1}\right]$. Let $\widehat{\operatorname{Mc}}(\mathcal{C})$ be the subgroup of $\operatorname{Out}(G)$ consisting of automorphisms leaving $\mathcal{C} \cup \mathcal{C}^{-1}$ globally invariant; it contains $\operatorname{Mc}(\mathcal{C})$ as a normal subgroup of finite index. We say that $\widehat{\operatorname{Mc}}(\mathcal{C})$ is an extended elementary McCool group of $G$.

More generally, if $\mathcal{H}$ is a finite family of subgroups, one can define finite extensions of $\operatorname{Mc}(\mathcal{H})$ by allowing the $H_{i}$ to be permuted or the action on $H_{i}$ to be only "almost" trivial.

Proposition 1.10 Given a toral relatively hyperbolic group $G$, there exists a number $C$ such that, if a subgroup $\widehat{M} \subset \operatorname{Out}(G)$ contains a group $\operatorname{Mc}(\mathcal{H})$ with finite index, then the index $[\widehat{M}: \operatorname{Mc}(\mathcal{H})]$ is bounded by $C$.

In particular, for $\mathcal{C}$ finite, the index of $\operatorname{Mc}(\mathcal{C})$ in $\widehat{\operatorname{Mc}}(\mathcal{C})$ is bounded by a constant depending only on $G$. 
It follows that extended elementary McCool groups satisfy a uniform chain condition as in Theorem 1.5 (see Corollary 6.4). We also have:

Corollary 1.11 Let $G$ be a toral relatively hyperbolic group. Let $A$ be any subgroup of $\operatorname{Out}(G)$ and let $\mathcal{C}_{A}$ be the (possibly infinite) set of conjugacy classes of $G$ whose $A$-orbit is finite. The image of $A$ in the group of permutations of $\mathcal{C}_{A}$ is finite and its order is bounded by a constant depending only on $G$. In other words, there is a subgroup $A_{0} \subset A$ of bounded finite index such that every conjugacy class in $G$ is fixed by $A_{0}$ or has infinite orbit under $A_{0}$.

When $G$ is free, one may take for $A_{0}$ the intersection of $A$ with a fixed finite-index subgroup of $\operatorname{Out}(G)$ (independent of $A$ ); see Handel and Mosher [26].

One may also consider subgroups of $\operatorname{Aut}(G)$.

Definition 1.12 Let $\mathcal{H}$ be a family of (conjugacy classes of) subgroups, and $H_{0}<G$ another subgroup. Let $\operatorname{Ac}\left(\mathcal{H}, H_{0}\right) \subset \operatorname{Aut}(G)$ be the group of automorphisms acting trivially on $\mathcal{H}$ (in the sense of Definition 1.2) and fixing the elements of $H_{0}$.

Proposition 1.13 If $G$ is a non-abelian, toral relatively hyperbolic group, then the group $\operatorname{Ac}\left(\mathcal{H}, H_{0}\right)$ is an extension

$$
1 \longrightarrow K \longrightarrow \operatorname{Ac}\left(\mathcal{H}, H_{0}\right) \longrightarrow \operatorname{Mc}\left(\mathcal{H}^{\prime}\right) \longrightarrow 1,
$$

where $\operatorname{Mc}\left(\mathcal{H}^{\prime}\right) \subset \operatorname{Out}(G)$ is a $\mathrm{McCool}$ group and $K$ is the centralizer of $H_{0}$ (isomorphic to $G$ or to $\mathbb{Z}^{n}$ for some $n \geq 0$ ).

Corollary 1.14 Theorems 1.3 and 1.5 also hold in $\operatorname{Aut}(G)$ : groups of the form $\operatorname{Ac}\left(\mathcal{H}, H_{0}\right)$ are of type VF and satisfy a uniform chain condition.

Theorems 1.3 and 1.4 are proved in Section 3 and Theorem 1.5 is proved in Section 5. All other results are proved in Section 6.

Acknowledgements Guirardel acknowledges support from ANR-11-BS01-013, the Institut Universitaire de France and the Lebesgue Center of Mathematics. Levitt acknowledges support from ANR-10-BLAN-116-03. 


\section{Preliminaries}

In this paper, $G$ will always denote a toral relatively hyperbolic group. Any non-trivial abelian subgroup $A$ of $G$ is contained in a unique maximal abelian subgroup. The maximal abelian subgroups are malnormal ( $G$ is CSA), finitely generated and there are finitely many non-cyclic ones up to conjugacy. Two subgroups of $A$ which are conjugate in $G$ are equal.

The center of a group $H$ will be denoted by $Z(H)$. We write $N_{K}(H)$ for the normalizer of a group $H$ in a group $K$, with $N(H)=N_{G}(H)$. Centralizers are called $Z_{K}(H)$.

We say that $\Phi \in \operatorname{Out}(G)$ preserves a subgroup $H$, or leaves $H$ invariant, if its representatives $\varphi \in \operatorname{Aut}(G)$ map $H$ to a conjugate. If $\varphi \in \operatorname{Aut}(G)$ equals the identity on $H$, we say that it fixes $H$.

Definition 2.1 If $\mathcal{H}$ is a family of subgroups, we let $\operatorname{Out}(G ; \mathcal{H}) \subset \operatorname{Out}(G)$ be the group of automorphisms preserving each $H \in \mathcal{H}$, and $\widehat{\operatorname{Out}}(G ; \mathcal{H})$ the group of automorphisms preserving $\mathcal{H}$ globally (possibly permuting groups in $\mathcal{H}$ ).

We denote by

$$
\operatorname{Out}\left(G ; \mathcal{H}^{(\mathrm{t})}\right)=\operatorname{Mc}(\mathcal{H}) \subset \operatorname{Out}(G)
$$

the group of automorphisms acting trivially on groups in $\mathcal{H}$ (as in Definition 1.2).

We write

$$
\begin{aligned}
\operatorname{Out}\left(G ; \mathcal{H}^{(\mathrm{t})}, \mathcal{K}\right) & :=\operatorname{Out}\left(G ; \mathcal{H}^{(\mathrm{t})}\right) \cap \operatorname{Out}(G ; \mathcal{K}), \\
\operatorname{Out}(G ; \mathcal{H}, \mathcal{K}) & :=\operatorname{Out}(G ; \mathcal{H} \cup \mathcal{K}) .
\end{aligned}
$$

Remark $\operatorname{Out}\left(G ; \mathcal{H}^{(\mathrm{t})}\right)$ and $\operatorname{Mc}(\mathcal{H})$ denote the same group. The notation $\operatorname{Out}\left(G ; \mathcal{H}^{(\mathrm{t})}\right)$ is more flexible and will be convenient in Section 3.

We will often view a set of conjugacy classes $\mathcal{C}=\left\{\left[c_{i}\right]\right\}$ as a family of cyclic subgroups $\mathcal{H}=\left\{\left\langle c_{i}\right\rangle\right\}$ since $\operatorname{Mc}(\mathcal{C})=\operatorname{Mc}(\mathcal{H})$. Note that $\operatorname{Out}(G ; \mathcal{H})$ is larger than $\operatorname{Mc}(\mathcal{C})=\operatorname{Mc}(\mathcal{H})$ since $c_{i}$ may sent to a conjugate of $c_{i}^{-1}$.

For example, suppose that $H<G=\mathbb{Z}^{n}$ is the subgroup generated by the first $k$ basis elements and $\mathcal{H}=\{H\}$. Then $\operatorname{Out}(G)=\operatorname{GL}(n, \mathbb{Z})$, the group $\operatorname{Out}(G ; \mathcal{H})$ consists of block triangular matrices, and $\operatorname{Out}\left(G ; \mathcal{H}^{(\mathrm{t})}\right)=\operatorname{Mc}(\mathcal{H})$ is the group of matrices fixing the first $k$ basis vectors.

There are inclusions $\operatorname{Out}\left(G ; \mathcal{H}^{(\mathrm{t})}\right) \subset \operatorname{Out}(G ; \mathcal{H}) \subset \widehat{\operatorname{Out}}(G ; \mathcal{H})$. Note that $\operatorname{Out}\left(G ; \mathcal{H}^{(\mathrm{t})}\right)$ has finite index in $\operatorname{Out}(G ; \mathcal{H})$ and $\widehat{\operatorname{Out}}(G ; \mathcal{H})$ if $\mathcal{H}$ is a finite family of cyclic groups. 
Given a family $\mathcal{H}$ and a subgroup $J$, we denote by $\mathcal{H}_{\mid J}$ the $J$-conjugacy classes of subgroups of $J$ conjugate to a group of $\mathcal{H}$. We view $\mathcal{H}_{\mid J}$ as a family of subgroups of $J$, each defined up to conjugacy in $J$. In the next subsection we will define a closely related notion $\mathcal{H}_{\| J}$ when $J=G_{v}$ is a vertex stabilizer in a tree.

If $\mathcal{C}$ is a set of conjugacy classes $\left[c_{i}\right]$, viewed as a set of cyclic subgroups, $\mathcal{C}_{\mid J}$ is the set of $J$-conjugacy classes of elements of $J$ representing elements in $\mathcal{C}$.

Now suppose that subgroups of $J$ which are conjugate in $G$ are conjugate in $J$; this holds for instance if $J$ is malnormal (in particular if $J$ is a free factor) and also if $J$ is abelian. In this case we may view $\mathcal{H}_{\mid J}$ as a subset of $\mathcal{H}$; it is finite if $\mathcal{H}$ is.

\subsection{Trees and splittings}

A tree will be a simplicial tree $T$ with an action of $G$ without inversions. A tree $T$ is relative to $\mathcal{H}$ (resp. $\mathcal{C}$ ) if any group in $\mathcal{H}$ (resp. any element representing a class in $\mathcal{C}$ ) fixes a point in $T$.

Two trees are considered to be the same if there is a $G$-equivariant isomorphism between them. In this paper, all trees will have abelian edge stabilizers.

Unless mentioned otherwise, we assume that the action is minimal (there is no proper invariant subtree). We usually assume that there is no redundant vertex (if $T \backslash\{x\}$ has two components, some $g \in G$ interchanges them). If a finitely generated subgroup $H \subset G$ acts on $T$ with no global fixed point, there is a smallest $H$-invariant subtree, called the minimal subtree of $H$.

The tree $T$ is trivial if there is a global fixed point (minimality then implies that $T$ is a point). An element or a subgroup of $G$ is elliptic if it fixes a point in $T$. Conjugates of elliptic subgroups are elliptic, so we also consider elliptic conjugacy classes.

An action of $G$ on a tree $T$ gives rise to a splitting of $G$, ie a decomposition of $G$ as the fundamental group of the quotient graph of groups $\Gamma=T / G$. Conversely, $T$ is the Bass-Serre tree of $\Gamma$. All definitions given here apply to both splittings and trees. In particular, a splitting is relative to $\mathcal{H}$ if every $H \in \mathcal{H}$ has a conjugate contained in a vertex group.

Minimality implies that the graph $\Gamma$ is finite. There is a one-to-one correspondence between vertices (resp. edges) of $\Gamma$ and $G$-orbits of vertices (resp. edges) of $T$. We denote by $V$ the set of vertices of $\Gamma$ and by $G_{v}$ the group carried by a vertex $v \in V$. We also view $v$ as a vertex of $T$ with stabilizer $G_{v}$. Similarly, we denote by $e$ an edge of $\Gamma$ or $T$, by $G_{e}$ the corresponding group (always abelian in this paper) and by $E$ the set of non-oriented edges of $\Gamma$. 
Edge groups being abelian, hence relatively quasiconvex, every vertex group $G_{v}$ is toral relatively hyperbolic (see for instance [25]).

The edge groups carried by edges of $\Gamma$ incident to a given vertex $v$ will be called the incident edge groups of $G_{v}$. We denote by $\operatorname{Inc}_{v}$ the family of incident edge groups (we view it as a finite family of subgroups of $G_{v}$, each well defined up to conjugacy).

If $\mathcal{H}$ is a finite family of subgroups of $G$ and $v$ is a vertex stabilizer of $T$, we denote by $\mathcal{H}_{\| G_{v}}$ the family of subgroups $H \subset G_{v}$ which are conjugate to a group of $\mathcal{H}$ and fix no other point in $T$. Two such groups are conjugate in $G_{v}$ if they are conjugate in $G$ (see [25, Lemma 2.2], where the notation $\mathcal{H}_{\mid G_{v}}$ is used instead), so we may also view $\mathcal{H}_{\| G_{v}}$ as a subset of $\mathcal{H}$ (it contains some of the groups of $\mathcal{H}$ having a conjugate in $G_{v}$ ), or as a finite family of subgroups of $G_{v}$, each well-defined up to conjugacy $\left(\mathcal{H}_{\| G_{v}}\right.$ may be smaller than $\mathcal{H}_{\mid G_{v}}$ because we do not include subgroups of edge groups).

Any splitting of $G_{v}$ relative to $\operatorname{Inc}_{v}$ extends to a splitting of $G$. If $T$ is relative to $\mathcal{H}$, any splitting of $G_{v}$ relative to $\operatorname{Inc}_{v} \cup \mathcal{H}_{\| G_{v}}$ is relative to $\mathcal{H}_{\mid G_{v}}$ and extends to a splitting of $G$ relative to $\mathcal{H}$.

If $\mathcal{C}$ is a set of conjugacy classes, we view $\mathcal{C}_{\| G_{v}}$ as the subset of $\mathcal{C}$ consisting of classes having a representative that fixes $v$ and no other vertex. In particular, $\mathcal{C}_{\| G_{v}}$ is finite if $\mathcal{C}$ is.

A tree $T^{\prime}$ is a collapse of $T$ if it is obtained from $T$ by collapsing each edge in a certain $G$-invariant collection to a point; conversely, we say that $T$ refines $T^{\prime}$. In terms of graphs of groups, one passes from $\Gamma=T / G$ to $\Gamma^{\prime}=T^{\prime} / G$ by collapsing edges; for each vertex $v^{\prime} \in \Gamma^{\prime}$, the vertex group $G_{v^{\prime}}$ is the fundamental group of the graph of groups $\Gamma_{v^{\prime}}$ occurring as the preimage of $v^{\prime}$ in $\Gamma$.

All maps between trees will be $G$-equivariant. Given two trees $T$ and $T^{\prime}$, we say that $T$ dominates $T^{\prime}$ if there is a map $f: T \rightarrow T^{\prime}$ or, equivalently, if every subgroup which is elliptic in $T$ is also elliptic in $T^{\prime}$; in particular, $T$ dominates any collapse $T^{\prime}$. We sometimes say that $f$ is a domination map. Minimality implies that it is onto.

Two trees belong to the same deformation space if they dominate each other. In other words, a deformation space $\mathcal{D}$ is the set of all trees having a given family of subgroups as their elliptic subgroups. We say that $\mathcal{D}$ dominates $\mathcal{D}^{\prime}$ if trees in $\mathcal{D}$ dominate those in $\mathcal{D}^{\prime}$.

\subsection{JSJ decompositions $[21 ; 22]$}

Let $\mathcal{H}$ be a family of subgroups of $G$. Recall that a tree $T$ is relative to $\mathcal{H}$ if all groups of $\mathcal{H}$ are elliptic in $T$. 
We denote by $\mathcal{H}^{+ \text {ab }}$ the family obtained by adding to $\mathcal{H}$ all non-cyclic abelian subgroups of $G$.

The group $G$ is freely indecomposable relative to $\mathcal{H}$ if it does not split over the trivial group relative to $\mathcal{H}$; equivalently, $G$ cannot be written non-trivially as $A * B$ with every group of $\mathcal{H}$ contained in a conjugate of $A$ or $B$ (if $\mathcal{H}$ is trivial, we also require $G \neq \mathbb{Z}$, as we consider $\mathbb{Z}$ as freely decomposable). Non-cyclic abelian groups being one-ended, being freely indecomposable relative to $\mathcal{H}$ is the same as being so relative to $\mathcal{H}^{+a b}$.

Let $\mathcal{A}$ be another family of subgroups (in this paper, $\mathcal{A}$ consists of the trivial group or is the family of all abelian subgroups). Once $\mathcal{H}$ and $\mathcal{A}$ are fixed, we only consider trees relative to $\mathcal{H}$, with edge stabilizers in $\mathcal{A}$. We also assume that trees are minimal.

A tree $T$ (with edge stabilizers in $\mathcal{A}$, relative to $\mathcal{H}$ ) is universally elliptic (with respect to $\mathcal{H}$ ) if its edge stabilizers are elliptic in every tree. It is a JSJ tree if, moreover, it dominates every universally elliptic tree. The set of JSJ trees is called the JSJ deformation space (over $\mathcal{A}$ relative to $\mathcal{H}$ ). All JSJ trees have the same vertex stabilizers, provided one restricts to stabilizers not in $\mathcal{A}$.

When $\mathcal{A}$ consists of the trivial group, the JSJ deformation space is called the Grushko deformation space (relative to $\mathcal{H}$ ). The group $G$ has a relative Grushko decomposition $G=G_{1} * \cdots * G_{n} * F_{p}$, with $F_{p}$ free, every $H \in \mathcal{H}$ contained in some $G_{i}$ (up to conjugacy) and $G_{i}$ freely indecomposable relative to $\mathcal{H}_{\mid G_{i}}$. Vertex stabilizers of the relative Grushko deformation space $\mathcal{D}$ are precisely conjugates of the $G_{i}$. The deformation space is trivial (it only contains the trivial tree) if and only if $G$ is freely indecomposable relative to $\mathcal{H}$. Writing $\mathcal{G}=\left\{G_{1}, \ldots, G_{n}\right\}$, note that $\operatorname{Out}(G ; \mathcal{H} \cup \mathcal{G})$ has finite index in $\operatorname{Out}(G ; \mathcal{H})$, because automorphisms in $\operatorname{Out}(G ; \mathcal{H})$ leave $\mathcal{D}$ invariant and therefore permute the $G_{i}$ (up to conjugacy).

Now suppose that $\mathcal{A}$ consists of all abelian subgroups and $G$ is freely indecomposable relative to a family $\mathcal{H}$. Then [22, Theorem 11.1$]$ the JSJ deformation space relative to $\mathcal{H}^{+\mathrm{ab}}$ contains a preferred tree $T_{\text {can }}$; this tree is invariant under $\widehat{\operatorname{Out}}(G ; \mathcal{H})$ (the group of automorphisms preserving $\mathcal{H}$ ).

It is obtained as a tree of cylinders. We describe this construction in the case that will be needed here (see [23, Proposition 6.3] for details). Let $T$ be any tree with non-trivial abelian edge stabilizers, relative to all non-cyclic abelian subgroups. Say that two edges $e$ and $e^{\prime}$ belong to the same cylinder if their stabilizers commute. Cylinders are subtrees intersecting in at most one point.

The tree of cylinders $T_{c}$ is defined as follows. It is bipartite, with vertex set $\mathcal{V}_{0} \cup \mathcal{V}_{1}$. Vertices in $\mathcal{V}_{0}$ are vertices of $T$ belonging to at least two cylinders. Vertices in $\mathcal{V}_{1}$ are cylinders of $T$. A vertex $v \in \mathcal{V}_{0}$ is joined to a vertex $Y \in \mathcal{V}_{1}$ if $v$ (viewed as a vertex 
of $T$ ) belongs to $Y$ (viewed as a subtree of $T$ ). Equivalently, one obtains $T_{c}$ from $T$ by replacing each cylinder $Y$ by the cone on its boundary (points of $Y$ belonging to at least one other cylinder).

The tree $T_{c}$ only depends on the deformation space $\mathcal{D}$ containing $T$ and it belongs to $\mathcal{D}$. Like $T$, it has non-trivial abelian edge stabilizers and is relative to all non-cyclic abelian subgroups. It is minimal if $T$ is minimal, but vertices in $\mathcal{V}_{1}$ may be redundant vertices.

The stabilizer of a vertex $v_{1} \in \mathcal{V}_{1}$ is a maximal abelian subgroup. The stabilizer of a vertex in $\mathcal{V}_{0}$ is non-abelian and is the stabilizer of a vertex of $T$. The stabilizer of an edge $v_{0} v_{1}$ with $v_{i} \in \mathcal{V}_{i}$ is an infinite abelian subgroup; it is a maximal abelian subgroup of $G_{v_{0}}$ (but it is not always maximal abelian in $G_{v_{1}}$ ).

The $\widehat{\operatorname{Out}}(G ; \mathcal{H})$-invariant tree $T_{\text {can }}$ mentioned above is the tree of cylinders of JSJ trees relative to $\mathcal{H}^{+\mathrm{ab}}$. It is a JSJ tree and the tree of cylinders of $T_{\text {can }}$ is $T_{\text {can }}$ itself.

Let $\Gamma_{\text {can }}=T_{\text {can }} / G$ be the quotient graph of groups and let $v \in \mathcal{V}_{0} / G$ be a vertex with $G_{v}$ non-abelian. If $G_{v}$ does not split over an abelian group relative to incident edge groups and to $\mathcal{H}_{\| G_{v}}$, it is universally elliptic (with respect to both $\mathcal{H}$ and $\mathcal{H}^{+ \text {ab }}$ ) and we say that $G_{v}$ (or $v$ ) is rigid; otherwise, it is flexible.

A key fact here is that every flexible vertex $v$ of $\Gamma_{\text {can }}$ is quadratically hanging $(Q H)$. The group $G_{v}$ is the fundamental group of a compact (possibly non-orientable) surface $\Sigma$, and incident edge groups are boundary subgroups of $\pi_{1}(\Sigma)$ (ie fundamental groups of boundary components of $\Sigma$ ); in particular, incident edge groups are cyclic. At most one incident edge group is attached to a given boundary component (groups carried by distinct incident edges are non-conjugate in $G_{v}$ ). If $H$ is conjugate to a group of $\mathcal{H}$, then $H \cap G_{v}$ is contained in a boundary subgroup. Conversely, every boundary subgroup is an incident edge group or has a finite-index subgroup which is conjugate to a group of $\mathcal{H}$.

As Szepietowski [34] does, we denote by $\mathcal{P} \mathcal{M}^{+}(\Sigma)$ the group of isotopy classes of homeomorphisms of $\Sigma$ mapping each boundary component to itself in an orientationpreserving way. We view $\mathcal{P} \mathcal{M}^{+}(\Sigma)$ as a subgroup of $\operatorname{Out}\left(\pi_{1}(\Sigma)\right)=\operatorname{Out}\left(G_{v}\right)$; indeed, $\mathcal{P} \mathcal{M}^{+}(\Sigma)=\operatorname{Out}\left(G_{v} ; \operatorname{Inc}_{v}^{(\mathrm{t})}, \mathcal{H}_{\| G_{v}}^{(\mathrm{t})}\right)$.

\subsection{Automorphisms of trees}

There is a natural action of $\operatorname{Out}(G)$ on the set of trees, given by precomposing the action on $T$ with an automorphism of $G$. We denote by $\operatorname{Out}(T)$ the stabilizer of a tree $T$. We write $\operatorname{Out}(T, \mathcal{H})$ for $\operatorname{Out}(T) \cap \operatorname{Out}(G ; \mathcal{H})$, and so on. 
If $T$ is a point, $\operatorname{Out}(T)=\operatorname{Out}(G)$. If $G$ is abelian and $T$ is not a point, then $T$ is a line on which $G$ acts by integral translations and $\operatorname{Out}(T)$ is the group of automorphisms of $G$ preserving the kernel of the action.

We now study $\operatorname{Out}(T)$ in the general case, following Levitt [27].

We always assume that edge stabilizers are abelian. This implies that all vertex or edge stabilizers $H$ have the property that the normalizer $N(H)$ acts on $H$ by inner automorphisms; indeed, $N(H)$ is abelian if $H$ is abelian and is equal to $H$ if $H$ is not abelian.

One first considers the action of $\operatorname{Out}(T)$ on the finite graph $\Gamma=T / G$. We always denote by $\operatorname{Out}^{0}(T)$ the finite-index subgroup consisting of automorphisms acting trivially.

We study it through the natural map

$$
\rho=\prod_{v \in V} \rho_{v}: \operatorname{Out}^{0}(T) \longrightarrow \prod_{v \in V} \operatorname{Out}\left(G_{v}\right)
$$

recording the action of automorphisms on vertex groups (see [27, Section 2]); recall that $V$ is the vertex set of $\Gamma$. Since $N\left(G_{v}\right)$ acts on $G_{v}$ by inner automorphisms, $\rho_{v}(\Phi)$ is simply defined as the class of $\alpha_{\mid G_{v}}$, where $\alpha \in \operatorname{Aut}(G)$ is any representative of $\Phi \in \mathrm{Out}^{0}(T)$ leaving $G_{v}$ invariant.

The image of $\rho$ is contained in $\prod_{v \in V} \operatorname{Out}\left(G_{v} ; \operatorname{Inc}_{v}\right.$ ) (the family of incident edge groups at a given $v$ is preserved). It contains the subgroup $\prod_{v \in V} \operatorname{Out}\left(G_{v} ; \operatorname{Inc}_{v}^{(t)}\right)$ because automorphisms of $G_{v}$ acting trivially on incident edge groups extend "by the identity" to automorphisms of $G$ preserving $T$.

The kernel of $\rho$ is the group of twists $\mathcal{T}$, a finitely generated abelian group when no edge group is trivial (bitwists as defined in [27] belong to $\mathcal{T}$ because the normalizer of an abelian subgroup is its centralizer). We therefore have an exact sequence

$$
1 \longrightarrow \mathcal{T} \longrightarrow \operatorname{Out}^{0}(T) \stackrel{\rho}{\rightarrow} \prod_{v \in V} \operatorname{Out}\left(G_{v} ; \operatorname{Inc}_{v}\right)
$$

Now suppose that $T$ is relative to families $\mathcal{H}$ and $\mathcal{K}$ (ie each $H_{i}$ and $K_{j}$ fixes a point in $T$ ). A trivial but important remark is that $\mathcal{T} \subset \operatorname{Out}\left(G ; \mathcal{H}^{(\mathrm{t})}, \mathcal{K}^{(\mathrm{t})}\right)$. As pointed out in [25, Lemma 2.10], we have

$$
\begin{aligned}
\prod_{v \in V} \operatorname{Out}\left(G_{v} ; \operatorname{Inc}_{v}^{(\mathrm{t})}, \mathcal{H}_{\| G_{v}}^{(\mathrm{t})}, \mathcal{K}_{\| G_{v}}\right) & \subset \rho\left(\operatorname{Out}^{0}(T) \cap \operatorname{Out}\left(G ; \mathcal{H}^{(\mathrm{t})}, \mathcal{K}\right)\right) \\
& \subset \prod_{v \in V} \operatorname{Out}\left(G_{v} ; \operatorname{Inc}_{v}, \mathcal{H}_{\| G_{v}}^{(\mathrm{t})}, \mathcal{K}_{\| G_{v}}\right)
\end{aligned}
$$


(see Section 2.1 for the definition of $\mathcal{H}_{\| G_{v}}$; groups of $\mathcal{H}_{\| G_{v}}$ that are conjugate in $G$ are necessarily conjugate in $G_{v}$ ).

The fact noted above that the image of $\operatorname{Out}^{0}(T)$ by $\rho$ contains $\prod_{v \in V} \operatorname{Out}\left(G_{v} ; \operatorname{Inc}_{v}^{(\mathrm{t})}\right)$ expresses that automorphisms $\Phi_{v} \in \operatorname{Out}\left(G_{v}\right)$ acting trivially on incident edge groups may be combined into a global $\Phi \in \operatorname{Out}(G)$. In Section 3.2.4 we will need a more general result, where we only assume that the $\Phi_{v}$ have compatible actions on edge groups.

Given an edge $e$ of $\Gamma$, there is a natural map $\rho_{e}$ : $\operatorname{Out}^{0}(T) \rightarrow \operatorname{Out}\left(G_{e}\right)$, defined in the same way as $\rho_{v}$ above. If $v$ is an endpoint of $e$, the inclusion of $G_{e}$ into $G_{v}$ induces a homomorphism $\rho_{v, e}: \operatorname{Out}\left(G_{v} ; \operatorname{Inc}_{v}\right) \rightarrow \operatorname{Out}\left(G_{e}\right)$ with $\rho_{e}=\rho_{v, e} \circ \rho_{v}$ (it is well-defined because the normalizer $N_{G_{v}}\left(G_{e}\right)$ acts on $G_{e}$ by inner automorphisms).

Lemma 2.2 Consider a family of automorphisms $\Phi_{v} \in \operatorname{Out}\left(G_{v}\right.$; Inc $\left.{ }_{v}\right)$ such that, if $e=v w$ is any edge of $\Gamma$, then $\rho_{v, e}\left(\Phi_{v}\right)=\rho_{w, e}\left(\Phi_{w}\right)$. There exists $\Phi \in \operatorname{Out}^{0}(T)$ such that $\rho_{v}(\Phi)=\Phi_{v}$ for every $v$.

We leave the proof to the reader. The lemma applies to any graph of groups such that, for every vertex or edge group $H$, the normalizer $N(H)$ acts on $H$ by inner automorphisms. $\Phi$ is not unique: it may be composed with any element of $\mathcal{T}$.

In Section 3.2.4 we will have a family of automorphisms $\Phi_{e} \in \operatorname{Out}\left(G_{e}\right)$ and we will want $\Phi \in \operatorname{Out}^{0}(T)$ such that $\rho_{e}(\Phi)=\Phi_{e}$ for every $e$. By the lemma, it suffices to find automorphisms $\Phi_{v} \in \operatorname{Out}\left(G_{v} ; \operatorname{Inc}_{v}\right)$ inducing the $\Phi_{e}$.

\subsection{Rigid vertices}

We now specialize to the case when $T=T_{\text {can }}$ is the canonical JSJ decomposition relative to $\mathcal{H}^{+\mathrm{ab}}$ discussed in Section 2.2.

If $v$ is a $\mathrm{QH}$ vertex, the image of $\operatorname{Out}^{0}(T) \cap \operatorname{Out}\left(G ; \mathcal{H}^{(\mathrm{t})}\right)$ in $\operatorname{Out}\left(G_{v}\right)$ contains $\mathcal{P} \mathcal{M}^{+}(\Sigma)=\operatorname{Out}\left(G_{v} ; \operatorname{Inc}_{v}^{(t)}, \mathcal{H}_{\| G_{v}}^{(t)}\right)$ with finite index (see [25, Proposition 4.7]).

If $v$ is a rigid vertex, then $G_{v}$ does not split over an abelian group relative to $\operatorname{Inc}_{v} \cup \mathcal{H}_{\| G_{v}}$. By the Bestvina-Paulin method and Rips theory, one deduces that the image of $\operatorname{Out}^{0}(T) \cap \operatorname{Out}\left(G ; \mathcal{H}^{(\mathrm{t})}\right)$ in $\operatorname{Out}\left(G_{v}\right)$ is finite if $\mathcal{H}$ is a finite family of finitely generated subgroups (see [25, Theorem 3.9 and Proposition 4.7]).

Lemma 2.3 Let $\mathcal{H}$ and $\mathcal{K}$ be finite families of finitely generated subgroups, with each group in $\mathcal{K}$ abelian. Assume that $G$ is one-ended relative to $\mathcal{H} \cup \mathcal{K}$ and let $T_{\text {can }}$ be the canonical $J S J$ tree relative to $(\mathcal{H} \cup \mathcal{K})^{\text {+ab }}$. 
The image of

$$
\operatorname{Out}^{0}(T) \cap \operatorname{Out}\left(G ; \mathcal{H}^{(\mathrm{t})}, \mathcal{K}\right)
$$

by $\rho_{v}: \operatorname{Out}^{0}(T) \rightarrow \operatorname{Out}\left(G_{v}\right)$ is finite if $v$ is a rigid vertex of $T_{\text {can }}$. Its image by $\rho_{e}: \operatorname{Out}^{0}(T) \rightarrow \operatorname{Out}\left(G_{e}\right)$ is finite if $e$ is any edge.

Proof Define $\mathcal{K}_{\mathbb{Z}}$ by removing all non-cyclic groups from $\mathcal{K}$. Being freely indecomposable relative to $\mathcal{H} \cup \mathcal{K}$ is the same as being freely indecomposable relative to $\mathcal{H} \cup \mathcal{K}_{\mathbb{Z}}$, and a tree is relative to $(\mathcal{H} \cup \mathcal{K})^{+a b}$ if and only if it is relative to $\left(\mathcal{H} \cup \mathcal{K}_{\mathbb{Z}}\right)^{+ \text {ab }}$. We may therefore view $T_{\text {can }}$ as the canonical JSJ tree relative to $\left(\mathcal{H} \cup \mathcal{K}_{\mathbb{Z}}\right)^{+ \text {ab }}$.

Let $v$ be a rigid vertex. The $\operatorname{group} \operatorname{Out}\left(G ; \mathcal{H}^{(\mathrm{t})}, \mathcal{K}\right)$ is contained in $\operatorname{Out}\left(G ; \mathcal{H}^{(\mathrm{t})}, \mathcal{K}_{\mathbb{Z}}\right)$, which contains $\operatorname{Out}\left(G ; \mathcal{H}^{(\mathrm{t})}, \mathcal{K}_{\mathbb{Z}}^{(\mathrm{t})}\right)$ with finite index. As explained above, the image of $\operatorname{Out}^{0}(T) \cap \operatorname{Out}\left(G ; \mathcal{H}^{(\mathrm{t})}, \mathcal{K}_{\mathbb{Z}}^{(\mathrm{t})}\right)$ in $\operatorname{Out}\left(G_{v}\right)$ is finite [25, Proposition 4.7]. The first assertion of the lemma follows.

Since $T_{\text {can }}$ is bipartite, every edge $e$ is incident to a vertex $v$ which is $\mathrm{QH}$ or rigid. In the first case $G_{e}$ is cyclic, so there is nothing to prove. In the second case the map $\rho_{e}: \operatorname{Out}^{0}(T) \rightarrow \operatorname{Out}\left(G_{e}\right)$ factors through $\operatorname{Out}\left(G_{v}\right)$ and the second assertion follows from the first.

\section{Finite classifying space}

In this section, we prove that McCool groups of a toral relatively hyperbolic group have type VF (Theorem 1.3) and that so does the stabilizer of a splitting (Theorem 1.4). In the course of the proof, we will describe the automorphisms of a given maximal abelian subgroup which are restrictions of an automorphism of $G$ belonging to a given McCool group (Proposition 3.10).

We start by recalling some standard facts about groups of type VF.

A group has type $\mathrm{F}$ if it has a finite classifying space and type VF if some finite-index subgroup is of type F. A key tool for proving that groups have type F is the following statement:

Theorem 3.1 (See for instance Geoghegan [15, Theorem 7.3.4]) Suppose that $G$ acts simplicially and cocompactly on a contractible simplicial complex $X$. If all point stabilizers have type $\mathrm{F}$, so does $G$. In particular, being of type $\mathrm{F}$ is stable under extensions.

If $G$ has a finite-index subgroup acting as in the theorem, then $G$ has type VF. In particular: 
Corollary 3.2 Given an exact sequence $1 \rightarrow N \rightarrow G \rightarrow Q \rightarrow 1$, suppose that $Q$ has type VF and $G$ has a finite-index subgroup $G_{0}<G$ such that $G_{0} \cap N$ has type F. Then $G$ has type VF.

Remark 3.3 Suppose that $G$ acts on $X$ as in Theorem 3.1. If point stabilizers are only of type VF, one cannot claim that $G$ has type VF, even if $G$ is torsion-free. This subtlety was overlooked in [20, Theorem 5.2] (we will give a corrected statement in Corollary 3.8) and it introduces technical complications (which would not occur if we only wanted to prove that the groups under consideration have type $\mathrm{F}_{\infty}$ ). In particular, to study the stabilizer of a tree with non-cyclic edge stabilizers in Section 3.2.3, we have to prove more precise versions of certain results (such as the "moreover" in Theorem 3.4).

\subsection{McCool groups are VF}

In this subsection we prove the following strengthening of Theorem 1.3:

Theorem 3.4 Let $G$ be a toral relatively hyperbolic group. Let $\mathcal{H}$ and $\mathcal{K}$ be two finite families of finitely generated subgroups, with each group in $\mathcal{K}$ abelian. Then $\operatorname{Out}\left(G ; \mathcal{H}^{(\mathrm{t})}, \mathcal{K}\right)$ is of type VF.

Moreover, if groups in $\mathcal{H}$ are also abelian, then there exists a finite-index subgroup $\operatorname{Out}^{1}(G ; \mathcal{H}, \mathcal{K}) \subset \operatorname{Out}(G ; \mathcal{H}, \mathcal{K})$ such that $\operatorname{Out}^{1}(G ; \mathcal{H}, \mathcal{K}) \cap \operatorname{Out}\left(G ; \mathcal{H}^{(\mathrm{t})}, \mathcal{K}\right)$ is of type $\mathrm{F}$.

Recall (Definition 2.1) that $\operatorname{Out}\left(G ; \mathcal{H}^{(\mathrm{t})}, \mathcal{K}\right)$ consists of classes of automorphisms acting trivially on each group $H_{i} \in \mathcal{H}$ (ie as conjugation by some $g_{i} \in G$ ) and leaving each $K_{j} \in \mathcal{K}$ invariant up to conjugacy.

It will follow from Corollary 1.6 that the main assertion of Theorem 3.4 holds if $\mathcal{H}$ is an arbitrary family of subgroups (see Corollary 6.3), but finiteness is needed at this point in order to apply Lemma 2.3.

Convention 3.5 In this subsection, a superscript 1 , as in $\operatorname{Out}^{1}(G ; \mathcal{H}, \mathcal{K})$, always indicates a subgroup of finite index. The superscript 0 refers to a trivial action on a quotient graph of groups (see Section 2.3).

3.1.1 The abelian case The following lemma deals with the case when $G=\mathbb{Z}^{n}$.

Lemma 3.6 Let $\mathcal{H}$ and $\mathcal{K}$ be finite families of subgroups of $\mathbb{Z}^{n}$. Consider the subgroup $A=\operatorname{Out}\left(\mathbb{Z}^{n} ; \mathcal{H}^{(\mathrm{t})}, \mathcal{K}\right)$ of $\mathrm{GL}(n, \mathbb{Z})$ consisting of matrices acting as the identity on groups $H_{i} \in \mathcal{H}$ and leaving each $K_{j} \in \mathcal{K}$ invariant. Then $A$ is of type VF. More precisely, every torsion-free subgroup of finite index $A^{\prime} \subset A$ is of type $\mathrm{F}$. 
Recall that $\operatorname{GL}(n, \mathbb{Z})$ is virtually torsion-free, so groups such as $A^{\prime}$ exist.

Proof The set of endomorphisms of $\mathbb{Z}^{n}$ acting as the identity on $H_{i}$ and preserving $K_{j}$ is a linear subspace defined by linear equations with rational coefficients. It follows that the groups $A$ and $A^{\prime}$ are arithmetic: they are commensurable with a subgroup of $\mathrm{GL}(n, \mathbb{Z})$ defined by $\mathbb{Q}$-linear equations. By Borel and Serre [7], every torsion-free arithmetic subgroup of $\operatorname{GL}(n, \mathbb{Q})$ is of type F.

To deduce Theorem 3.4 when $G$ is abelian, we simply define $\operatorname{Out}^{1}(G ; \mathcal{H}, \mathcal{K})$ as any torsion-free, finite-index subgroup of $\operatorname{Out}(G ; \mathcal{H}, \mathcal{K})$.

If $G$ is not abelian, we shall distinguish two cases.

3.1.2 The one-ended case We first assume that $G$ is freely indecomposable relative to $\mathcal{H} \cup \mathcal{K}$ : one cannot write $G=A * B$ with each group of $\mathcal{H} \cup \mathcal{K}$ contained in a conjugate of $A$ or $B$. We then consider the canonical tree $T_{\text {can }}$ as in Section 2.2 (it is a JSJ tree relative to $\mathcal{H}, \mathcal{K}$ and to non-cyclic abelian subgroups). It is invariant under $\operatorname{Out}(G ; \mathcal{H}, \mathcal{K})$, so $\operatorname{Out}(G ; \mathcal{H}, \mathcal{K}) \subset \operatorname{Out}\left(T_{\text {can }}\right)$.

We write $\operatorname{Out}^{0}\left(T_{\text {can }}\right)$ for the finite-index subgroup consisting of automorphisms acting trivially on the finite graph $\Gamma_{\text {can }}=T_{\text {can }} / G$ and

$$
\operatorname{Out}^{0}(G ; \mathcal{H}, \mathcal{K})=\operatorname{Out}(G ; \mathcal{H}, \mathcal{K}) \cap \operatorname{Out}^{0}\left(T_{\text {can }}\right),
$$

which has finite index in $\operatorname{Out}(G ; \mathcal{H}, \mathcal{K})$.

Recall that non-abelian vertex stabilizers $G_{v}$ of $T_{\text {can }}$ (or vertex groups of $\Gamma_{\text {can }}$ ) are rigid or $\mathrm{QH}$. Also recall from Section 2.3 that, for each vertex $v$, there is a map $\rho_{v}: \operatorname{Out}^{0}\left(T_{\text {can }}\right) \rightarrow \operatorname{Out}\left(G_{v} ; \operatorname{Inc}_{v}\right)$, with $\operatorname{Inc}_{v}$ the family of incident edge groups (see Section 2.1).

We define a subgroup $\operatorname{Out}^{r}(G ; \mathcal{H}, \mathcal{K}) \subset \operatorname{Out}(G ; \mathcal{H}, \mathcal{K})$ by restricting to automorphisms $\Phi \in \operatorname{Out}^{0}(G ; \mathcal{H}, \mathcal{K})$ and imposing conditions on the image of $\Phi$ by the maps $\rho_{v}$ :

- If $G_{v}$ is rigid, we ask that $\rho_{v}(\Phi)$ be trivial.

- If $G_{v}$ is abelian, we fix a torsion-free subgroup of finite index $\operatorname{Out}^{1}\left(G_{v}\right) \subset \operatorname{Out}\left(G_{v}\right)$ and we ask that $\rho_{v}(\Phi)$ belong to $\operatorname{Out}^{1}\left(G_{v}\right)$.

- If $G_{v}$ is $\mathrm{QH}$, it is the fundamental group of a compact surface $\Sigma$. Each boundary component is associated to an incident edge or a group in $\mathcal{H} \cup \mathcal{K}$ (see Section 2.2), so $\rho_{v}(\Phi)$ preserves the peripheral structure of $\pi_{1}(\Sigma)$ and may therefore be represented by a homeomorphism of $\Sigma$. Since groups in $\mathcal{H} \cup \mathcal{K}$, and their conjugates, only intersect $G_{v}$ 
along boundary subgroups, the image of $\operatorname{Out}^{0}(G ; \mathcal{H}, \mathcal{K})$ by $\rho_{v}$ contains the mapping class group

$$
\mathcal{P} \mathcal{M}^{+}(\Sigma)=\operatorname{Out}\left(G_{v} ; \operatorname{Inc}_{v}^{(\mathrm{t})}, \mathcal{H}_{\| G_{v}}^{(\mathrm{t})}, \mathcal{K}_{\| G_{v}}^{(\mathrm{t})}\right)
$$

(see Section 2.2); the index is finite. We fix a finite-index subgroup $\mathcal{P} \mathcal{M}^{+, 1}(\Sigma)$ of type $\mathrm{F}$ and we require $\rho_{v}(\Phi) \in \mathcal{P} \mathcal{M}^{+, 1}(\Sigma)$. In particular, $\Phi$ acts trivially on all boundary subgroups of $\Sigma$.

Let $\operatorname{Out}^{r}(G ; \mathcal{H}, \mathcal{K})$ consist of automorphisms $\Phi \in \operatorname{Out}^{0}(G ; \mathcal{H}, \mathcal{K})$ whose images $\rho_{v}(\Phi)$ satisfy the above conditions. These automorphisms act trivially on edge stabilizers.

It follows from Lemma 2.3 that $\operatorname{Out}^{r}(G ; \mathcal{H}, \mathcal{K}) \cap \operatorname{Out}\left(G ; \mathcal{H}^{(\mathrm{t})}, \mathcal{K}\right)$ always has finite index in $\operatorname{Out}\left(G ; \mathcal{H}^{(\mathrm{t})}, \mathcal{K}\right)$. If groups in $\mathcal{H}$ are abelian, then $\operatorname{Out}^{r}(G ; \mathcal{H}, \mathcal{K})$ has finite index in $\operatorname{Out}(G ; \mathcal{H}, \mathcal{K})$. It therefore suffices to prove that

$$
O:=\operatorname{Out}^{r}(G ; \mathcal{H}, \mathcal{K}) \cap \operatorname{Out}\left(G ; \mathcal{H}^{(\mathrm{t})}, \mathcal{K}\right)
$$

is of type $\mathrm{F}$ (this argument, based on Lemma 2.3, is the only place where we use the assumptions on $\mathcal{H}$ and $\mathcal{K}$ ).

Every edge of $T_{\text {can }}$ has an endpoint $v$ with $G_{v}$ rigid or $\mathrm{QH}$, so elements of $O$ act trivially on edge stabilizers of $T_{\text {can }}$. Consider an abelian vertex stabilizer $G_{v}$. Elements in $\rho_{v}(O)$ are the identity on incident edge groups and groups in $\mathcal{H}_{\| G_{v}}$, and leave groups in $\mathcal{K}_{\| G_{v}}$ invariant. By Lemma 3.6 these conditions define a group $B_{v} \subset \operatorname{Out}\left(G_{v}\right)$ which is of type VF and $C_{v}:=B_{v} \cap \operatorname{Out}^{1}\left(G_{v}\right)$ is a group of type F containing $\rho_{v}(O)$.

Recall from Section 2.3 the exact sequence

$$
1 \longrightarrow \mathcal{T} \longrightarrow \operatorname{Out}^{0}\left(T_{\text {can }}\right) \stackrel{\rho}{\rightarrow} \prod_{v \in V} \operatorname{Out}\left(G_{v} ; \operatorname{Inc}_{v}\right)
$$

We claim that the image of $O$ by $\rho$ is a direct product $\prod_{v \in V} C_{v}$, with $C_{v}$ as above if $G_{v}$ is abelian, $C_{v}=\mathcal{P} \mathcal{M}^{+, 1}(\Sigma)$ if $v$ is $\mathrm{QH}$, and $C_{v}$ trivial if $v$ is rigid. The image is contained in the product. Conversely, given a family $\left(\Phi_{v}\right)_{v \in V}$, with $\Phi_{v} \in C_{v}$, the automorphisms $\Phi_{v}$ act trivially on incident edge groups, so there is $\Phi \in \operatorname{Out}^{0}\left(T_{\text {can }}\right)$ with $\rho_{v}(\Phi)=\Phi_{v}$. Since $\Phi_{v}$ acts trivially on $\operatorname{Inc}_{v} \cup \mathcal{H}_{\| G_{v}}$ and preserves $\mathcal{K}_{\| G_{v}}$, this automorphism is in $O$. This proves the claim.

It follows that $\rho(O)$ is of type $\mathrm{F}$. The group of twists $\mathcal{T}$ is contained in $O$, because twists act trivially on vertex groups and $T$ is relative to $\mathcal{H} \cup \mathcal{K}$, so we can conclude that $O$ is of type $\mathrm{F}$ by Theorem 3.1 if we know that $\mathcal{T}$ is of type F. The group $\mathcal{T}$ is a finitely generated abelian group. It is torsion-free, hence of type $\mathrm{F}$, as shown in [25, Section 4] (alternatively, one can replace $\operatorname{Out}^{r}(G ; \mathcal{H}, \mathcal{K})$ by its intersection with a torsion-free, finite-index subgroup of $\operatorname{Out}(G)$, which exists by [25, Corollary 4.4]). 
This proves Theorem 3.4 in the freely indecomposable case. To prove it in general, we need to study automorphisms of free products.

3.1.3 Automorphisms of free products In this subsection, $G$ does not have to be relatively hyperbolic.

Let $\mathcal{G}=\left\{G_{i}\right\}$ be a family of subgroups of $G$. We have defined $\operatorname{Out}(G ; \mathcal{G})$ as automorphisms leaving the conjugacy class of each $G_{i}$ invariant and $\operatorname{Out}\left(G ; \mathcal{G}^{(\mathrm{t})}\right)$ as automorphisms acting trivially on each $G_{i}$.

More generally, consider a group of automorphisms $\mathcal{Q}_{i} \subset \operatorname{Out}\left(G_{i}\right)$ and $\mathcal{Q}=\left\{\mathcal{Q}_{i}\right\}$. We would like to define $\operatorname{Out}\left(G ; \mathcal{G}^{(\mathcal{Q})}\right) \subset \operatorname{Out}(G ; \mathcal{G})$ as the automorphisms $\Phi$ acting on each $G_{i}$ as an element of $\mathcal{Q}_{i}$. To be precise, given $\Phi \in \operatorname{Out}(G ; \mathcal{G})$, choose representatives $\varphi_{i}$ of $\Phi$ in $\operatorname{Aut}(G)$ with $\varphi_{i}\left(G_{i}\right)=G_{i}$. We say that $\Phi$ belongs to $\operatorname{Out}\left(G ; \mathcal{G}^{(\mathcal{Q})}\right)$ if every $\varphi_{i}$ represents an element of $\mathcal{Q}_{i}$. This is well-defined (independent of the chosen $\varphi_{i}$ ) if each $G_{i}$ is a free factor (more generally, if the normalizer of $G_{i}$ acts on $G_{i}$ by inner automorphisms).

The goal of this subsection is to show:

Proposition 3.7 Let $G=G_{1} * \cdots * G_{n} * F_{p}$, with $F_{p}$ free of rank $p$, and let $\mathcal{G}=\left\{G_{i}\right\}$. Assume that all groups $G_{i}$ and $G_{i} / Z\left(G_{i}\right)$ have type $\mathrm{F}$.

Let $\mathcal{Q}=\left\{\mathcal{Q}_{i}\right\}$ be a family of subgroups $\mathcal{Q}_{i} \subset \operatorname{Out}\left(G_{i}\right)$. If every $\mathcal{Q}_{i}$ is of type VF, then $\operatorname{Out}\left(G ; \mathcal{G}^{(\mathcal{Q})}\right)$ has type VF.

More precisely, there exists a finite-index subgroup $\operatorname{Out}^{1}(G ; \mathcal{G}) \subset \operatorname{Out}(G ; \mathcal{G})$, independent of $\mathcal{Q}$, such that, if every $\mathcal{Q}_{i}$ is of type $\mathrm{F}$, then $\operatorname{Out}^{1}(G ; \mathcal{G}) \cap \operatorname{Out}\left(G ; \mathcal{G}^{(\mathcal{Q})}\right)$ has type F.

The "more precise" assertion implies the first one, since $\operatorname{Out}\left(G ; \mathcal{G}^{\left(\mathcal{Q}^{\prime}\right)}\right)$ has finite index in $\operatorname{Out}\left(G ; \mathcal{G}^{(\mathcal{Q})}\right)$ if every $\mathcal{Q}_{i}^{\prime}$ is a finite-index subgroup of $\mathcal{Q}_{i}$.

Assume that $G_{i}$ and $G_{i} / Z\left(G_{i}\right)$ have type F. The proposition says in particular that the Fouxe-Rabinovitch group $\operatorname{Out}\left(G ; \mathcal{G}^{(\mathrm{t})}\right)$ is of type VF, and that $\operatorname{Out}(G ; \mathcal{G})$ is of type VF if every $\operatorname{Out}\left(G_{i}\right)$ is. If we consider the Grushko decomposition of $G$, then $\operatorname{Out}(G ; \mathcal{G})$ has finite index in $\operatorname{Out}(G)$ and we get:

Corollary 3.8 (Correcting [20, Theorem 5.2]) Let $G=G_{1} * \cdots * G_{n} * F_{p}$, with $F_{p}$ free and $G_{i}$ non-trivial, not isomorphic to $\mathbb{Z}$ and not a free product. If every $G_{i}$ and $G_{i} / Z\left(G_{i}\right)$ has type $\mathrm{F}$ and every $\operatorname{Out}\left(G_{i}\right)$ has type $\mathrm{VF}$, then $\operatorname{Out}(G)$ has type VF. 
Proof of Proposition 3.7 We prove the "more precise" assertion, so we assume that $\mathcal{Q}_{i} \subset \operatorname{Out}\left(G_{i}\right)$ has type F. We shall apply Theorem 3.1 to the $\operatorname{action}$ of $\operatorname{Out}\left(G ; \mathcal{G}^{(\mathcal{Q})}\right)$ on the outer space defined in [20]. We let $\mathcal{D}$ be the Grushko deformation space relative to $\mathcal{G}$, ie the JSJ deformation space of $G$ over the trivial group relative to $\mathcal{G}$ (see Section 2.2). Trees in $\mathcal{D}$ have trivial edge stabilizers and non-trivial vertex stabilizers are conjugates of the $G_{i}$.

Like ordinary outer space [9], the projectivization $\widehat{\mathcal{D}}$ of $\mathcal{D}$ is a complex consisting of simplices with missing faces and the spine of $\widehat{\mathcal{D}}$ is a simplicial complex. It is contractible for the weak topology [19].

The group $\operatorname{Out}(G ; \mathcal{G})$ acts on $\mathcal{D}$, hence on the spine, and the action of the FouxeRabinovitch group $\operatorname{Out}\left(G ; \mathcal{G}^{(\mathrm{t})}\right) \subset \operatorname{Out}\left(G ; \mathcal{G}^{(\mathcal{Q})}\right)$ is cocompact because there are finitely many possibilities for the quotient graph $T / G$ for $T \in \mathcal{D}$. In order to apply Theorem 3.1, we just need to show that stabilizers are of type $\mathrm{F}$.

$\operatorname{Out}(G ; \mathcal{G})$ also acts on the free group (isomorphic to $F_{p}$ ) obtained from $G$ by killing all the $G_{i}$ (it may be viewed as the topological fundamental group of $\Gamma=T / G$ for any $T \in \mathcal{D})$. In other words, there is a natural map $\operatorname{Out}(G ; \mathcal{G}) \rightarrow \operatorname{Out}\left(F_{p}\right)$. We fix a torsion-free, finite-index subgroup $\operatorname{Out}^{1}\left(F_{p}\right) \subset \operatorname{Out}\left(F_{p}\right)$ and we define $\operatorname{Out}^{1}(G ; \mathcal{G}) \subset \operatorname{Out}(G ; \mathcal{G})$ as the pullback of $\operatorname{Out}^{1}\left(F_{p}\right)$.

Given $T \in \mathcal{D}$, we let $S$ be its stabilizer for the action of $\operatorname{Out}^{1}(G ; \mathcal{G})$ and $S_{\mathcal{Q}}$ its stabilizer for the action of $\operatorname{Out}^{1}(G ; \mathcal{G}) \cap \operatorname{Out}\left(G ; \mathcal{G}^{(\mathcal{Q})}\right)$. We complete the proof by showing that $S_{\mathcal{Q}}$ has type F.

We first claim that $S$ equals $\operatorname{Out}^{0}(T)$, the group of automorphisms of $G$ leaving $T$ invariant and acting trivially on $\Gamma=T / G$. Clearly $\operatorname{Out}^{0}(T) \subset S$. Conversely, we have to show that any $\Phi \in S$ acts as the identity on $\Gamma$. First, $\Phi$ fixes all vertices of $\Gamma$ carrying a non-trivial group $G_{v}$, because $G_{v}$ is a $G_{i}$ (up to conjugacy) and the $G_{i}$ are not permuted. In particular, by minimality of $T$, all terminal vertices of $\Gamma$ are fixed. Also, by our definition of $\operatorname{Out}^{1}(G ; \mathcal{G})$, the image of $\Phi$ in $\operatorname{Out}\left(\pi_{1}(\Gamma)\right)$ is trivial or has infinite order. The claim follows because any non-trivial symmetry of $\Gamma$ fixing all terminal vertices maps to a non-trivial element of finite order in $\operatorname{Out}\left(\pi_{1}(\Gamma)\right)$ if $\Gamma$ is not a circle. The map $\rho$ (see Section 2.3) maps $S$ onto $\prod_{i} \operatorname{Out}\left(G_{i}\right)$, and the image of $S_{\mathcal{Q}}$ is $\prod_{i} \mathcal{Q}_{i}$, a group of type $\mathrm{F}$. The kernel is the group of twists $\mathcal{T}$, which is contained in $S_{\mathcal{Q}}$, so it suffices to check that $\mathcal{T}$ has type F. Since edge stabilizers are trivial, $\mathcal{T}$ is a direct product $\prod_{i} K_{i}$, with $K_{i}=G_{i}^{n_{i}} / Z\left(G_{i}\right)$; here $n_{i}$ is the valence of the vertex carrying $G_{i}$ in $\Gamma$ and the center $Z\left(G_{i}\right)$ is embedded diagonally (see [27]). There are exact sequences

$$
1 \longrightarrow G_{i}^{n_{i}-1} \longrightarrow G_{i}^{n_{i}} / Z\left(G_{i}\right) \longrightarrow G_{i} / Z\left(G_{i}\right) \longrightarrow 1,
$$

so the assumptions of the proposition ensure that $\mathcal{T}$ is of type $\mathrm{F}$. 
3.1.4 The infinitely ended case We can now prove Theorem 3.4 in full generality. We let $G=G_{1} * \cdots * G_{n} * F_{p}$ be the Grushko decomposition of $G$ relative to $\mathcal{H} \cup \mathcal{K}$ (see Section 2.2) and $\mathcal{G}=\left\{G_{i}\right\}$. Each $G_{i}$ is toral relatively hyperbolic, so has type $\mathrm{F}$ by Dahmani [10]. Its center is trivial if $G_{i}$ is nonabelian, so $G_{i} / Z\left(G_{i}\right)$ also has type F. This will allow us to use Proposition 3.7.

Lemma 3.9 Let $\mathcal{Q}=\left\{\mathcal{Q}_{i}\right\}$ with $\mathcal{Q}_{i}=\operatorname{Out}\left(G_{i} ; \mathcal{H}_{\mid G_{i}}^{(\mathrm{t})}, \mathcal{K}_{\mid G_{i}}\right)$ and let $\mathcal{R}=\left\{\mathcal{R}_{i}\right\}$ with $\mathcal{R}_{i}=\operatorname{Out}\left(G_{i} ; \mathcal{H}_{\mid G_{i}}, \mathcal{K}_{\mid G_{i}}\right)$. Then

$$
\begin{aligned}
& \operatorname{Out}\left(G ; \mathcal{G}^{(\mathcal{Q})}\right)=\operatorname{Out}\left(G ; \mathcal{H}^{(\mathrm{t})}, \mathcal{K}\right) \cap \operatorname{Out}(G ; \mathcal{G}), \\
& \operatorname{Out}\left(G ; \mathcal{G}^{(\mathcal{R})}\right)=\operatorname{Out}(G ; \mathcal{H}, \mathcal{K}) \cap \operatorname{Out}(G ; \mathcal{G}) .
\end{aligned}
$$

Moreover, $\operatorname{Out}\left(G ; \mathcal{G}^{(\mathcal{Q})}\right)$ has finite index in $\operatorname{Out}\left(G ; \mathcal{H}^{(\mathrm{t})}, \mathcal{K}\right)$ and $\operatorname{Out}\left(G ; \mathcal{G}^{(\mathcal{R})}\right)$ has finite index in $\operatorname{Out}(G ; \mathcal{H}, \mathcal{K})$.

Proof If $\Phi$ belongs to $\operatorname{Out}\left(G ; \mathcal{G}^{(\mathcal{Q})}\right)$, it belongs to $\operatorname{Out}\left(G ; \mathcal{H}^{(\mathrm{t})}, \mathcal{K}\right)$, because every group in $\mathcal{H} \cup \mathcal{K}$ has a conjugate contained in some $G_{i}$. Conversely, automorphisms in $\operatorname{Out}\left(G ; \mathcal{H}^{(\mathrm{t})}, \mathcal{K}\right)$ preserve the Grushko deformation space relative to $\mathcal{H} \cup \mathcal{K}$ and therefore permute the $G_{i}$, so $\operatorname{Out}(G ; \mathcal{G}) \cap \operatorname{Out}\left(G ; \mathcal{H}^{(\mathrm{t})}, \mathcal{K}\right)$ has finite index in $\operatorname{Out}\left(G ; \mathcal{H}^{(\mathrm{t})}, \mathcal{K}\right)$. If $\varphi \in \operatorname{Aut}(G)$ leaves $G_{i}$ invariant and maps a non-trivial $H \subset G_{i}$ to a conjugate $\mathrm{gHg}^{-1}$, then $g \in G_{i}$ because $G_{i}$ is a free factor. This shows

$$
\operatorname{Out}\left(G ; \mathcal{H}^{(\mathrm{t})}, \mathcal{K}\right) \cap \operatorname{Out}(G ; \mathcal{G}) \subset \operatorname{Out}\left(G ; \mathcal{G}^{(\mathcal{Q})}\right),
$$

completing the proof for $\operatorname{Out}\left(G ; \mathcal{G}^{(\mathcal{Q})}\right)$. The proof for $\operatorname{Out}\left(G ; \mathcal{G}^{(\mathcal{R})}\right)$ is similar.

The first assertion of Theorem 3.4 now follows immediately from the one-ended case together with Proposition 3.7, $\operatorname{since} \operatorname{Out}\left(G ; \mathcal{H}^{(\mathrm{t})}, \mathcal{K}\right)$ contains $\operatorname{Out}\left(G ; \mathcal{G}^{(\mathcal{Q})}\right)$ with finite index. There remains to prove the "moreover".

Each $G_{i}$ is freely indecomposable relative to $\mathcal{H}_{\mid G_{i}} \cup \mathcal{K}_{\mid G_{i}}$, so we may apply the "moreover" of Theorem 3.4 to $G_{i}$. We get a finite-index subgroup $\mathcal{R}_{i}^{1} \subset \mathcal{R}_{i}$ such that $\mathcal{Q}_{i}^{1}:=\mathcal{R}_{i}^{1} \cap \mathcal{Q}_{i}$ has type F. Let $\mathcal{R}^{1}=\left\{\mathcal{R}_{i}^{1}\right\}$ and $\mathcal{Q}^{1}=\left\{\mathcal{Q}_{i}^{1}\right\}$.

By Proposition 3.7, there is a finite-index subgroup $\operatorname{Out}^{1}(G ; \mathcal{G}) \subset \operatorname{Out}(G ; \mathcal{G})$ such that $\operatorname{Out}^{1}(G ; \mathcal{G}) \cap \operatorname{Out}\left(G ; \mathcal{G}^{\left(\mathcal{Q}^{1}\right)}\right)$ has type F. Now write

$\operatorname{Out}^{1}(G ; \mathcal{G}) \cap \operatorname{Out}\left(G ; \mathcal{G}^{\left(\mathcal{Q}^{1}\right)}\right)=\operatorname{Out}^{1}(G ; \mathcal{G}) \cap \operatorname{Out}\left(G ; \mathcal{G}^{\left(\mathcal{R}^{1}\right)}\right) \cap \operatorname{Out}\left(G ; \mathcal{G}^{(\mathcal{Q})}\right)$.

By Lemma 3.9, we may replace the last term $\operatorname{Out}\left(G ; \mathcal{G}^{(\mathcal{Q})}\right)$ by $\operatorname{Out}\left(G ; \mathcal{H}^{(\mathrm{t})}, \mathcal{K}\right)$. Defining

$$
\operatorname{Out}^{1}(G ; \mathcal{H}, \mathcal{K}):=\operatorname{Out}^{1}(G ; \mathcal{G}) \cap \operatorname{Out}\left(G ; \mathcal{G}^{\left(\mathcal{R}^{1}\right)}\right)
$$


we have shown that $\operatorname{Out}^{1}(G ; \mathcal{H}, \mathcal{K}) \cap \operatorname{Out}\left(G ; \mathcal{H}^{(\mathrm{t})}, \mathcal{K}\right)$ has type $\mathrm{F}$. There remains to check that $\operatorname{Out}^{1}(G ; \mathcal{H}, \mathcal{K})$ is a finite-index subgroup of $\operatorname{Out}(G ; \mathcal{H}, \mathcal{K})$.

Since $\operatorname{Out}^{1}(G ; \mathcal{G})$ has finite index $\operatorname{in} \operatorname{Out}(G ; \mathcal{G})$ and $\mathcal{R}_{i}^{1}$ is a finite-index subgroup of $\mathcal{R}_{i}$, the group $\operatorname{Out}^{1}(G ; \mathcal{H}, \mathcal{K})$ has finite index in $\operatorname{Out}(G ; \mathcal{G}) \cap \operatorname{Out}\left(G ; \mathcal{G}^{(\mathcal{R})}\right)$, which equals $\operatorname{Out}\left(G ; \mathcal{G}^{(\mathcal{R})}\right)$ and has finite index in $\operatorname{Out}(G ; \mathcal{H}, \mathcal{K})$ by Lemma 3.9.

This completes the proof of Theorem 3.4.

3.1.5 The action on abelian groups We study the action of $\operatorname{Out}(G)$ on abelian subgroups. The result of this subsection (Proposition 3.10) will be needed in Section 3.2.4.

A toral relatively hyperbolic group has finitely many conjugacy classes of non-cyclic maximal abelian subgroups. Fix a representative $A_{j}$ in each class. Automorphisms of $G$ preserve the set of $A_{j}$ (up to conjugacy), so some finite-index subgroup of $\operatorname{Out}(G)$ maps to $\prod_{j} \operatorname{Out}\left(A_{j}\right)$. We shall show in particular that the image of a suitable finite-index subgroup $\operatorname{Out}^{\prime}(G) \subset \operatorname{Out}(G)$ is a product of McCool groups $\prod_{j} \operatorname{Out}\left(A_{j} ;\left\{F_{j}\right\}^{(\mathrm{t})}\right) \subset$ $\prod_{j} \operatorname{Out}\left(A_{j}\right)$.

This product structure expresses the fact that automorphisms of non-conjugate maximal non-cyclic abelian subgroups do not interact. Indeed, consider a family of elements $\Phi_{j} \in \operatorname{Out}\left(A_{j}\right)$ and suppose that each $\Phi_{j}$, taken individually, extends to an automorphism $\widehat{\Phi}_{j} \in \operatorname{Out}^{\prime}(G)$; then there is $\Phi \in \operatorname{Out}^{\prime}(G)$ inducing all $\Phi_{j}$ simultaneously.

In fact, we will work with two (possibly empty) finite families $\mathcal{H}$ and $\mathcal{K}$ of abelian subgroups and we will restrict to $\operatorname{Out}\left(G ; \mathcal{H}^{(\mathrm{t})}, \mathcal{K}\right)$. We shall therefore define a finiteindex subgroup $\operatorname{Out}^{\prime}\left(G ; \mathcal{H}^{(\mathrm{t})}, \mathcal{K}\right) \subset \operatorname{Out}\left(G ; \mathcal{H}^{(\mathrm{t})}, \mathcal{K}\right)$.

First assume that $G$ is freely indecomposable relative to $\mathcal{H} \cup \mathcal{K}$. As in Section 3.1.2, we consider the canonical JSJ tree $T_{\text {can }}$, we restrict to automorphisms $\Phi \in \operatorname{Out}\left(G ; \mathcal{H}^{(\mathrm{t})}, \mathcal{K}\right)$ acting trivially on $\Gamma_{\text {can }}=T_{\text {can }} / G$ and we define $\operatorname{Out}^{\prime}\left(G ; \mathcal{H}^{(\mathrm{t})}, \mathcal{K}\right)$ by imposing conditions on the action on non-abelian vertex groups $G_{v}$ : if $G_{v}$ is $\mathrm{QH}$, the action should be trivial on all boundary subgroups of $\Sigma$ (ie $\rho_{v}(\Phi) \in \mathcal{P} \mathcal{M}^{+}(\Sigma)$ ); if $G_{v}$ is rigid, then $\rho_{v}(\Phi)$ should be trivial. We have explained in Section 3.1.2 why this defines a subgroup of finite index $\operatorname{Out}^{\prime}\left(G ; \mathcal{H}^{(\mathrm{t})}, \mathcal{K}\right)$ in $\operatorname{Out}\left(G ; \mathcal{H}^{(\mathrm{t})}, \mathcal{K}\right)$. Note that $\operatorname{Out}^{\prime}\left(G ; \mathcal{H}^{(\mathrm{t})}, \mathcal{K}\right)$ acts trivially on edge groups of $T_{\text {can }}$.

If $G$ is not freely indecomposable relative to $\mathcal{H} \cup \mathcal{K}$, let $G=G_{1} * \cdots * G_{n} * F_{p}$ be the relative Grushko decomposition. To define $\operatorname{Out}^{\prime}\left(G ; \mathcal{H}^{(\mathrm{t})}, \mathcal{K}\right)$, we require that $\Phi$ maps $G_{i}$ to $G_{i}$ (up to conjugacy) and the induced automorphism belongs to $\operatorname{Out}^{\prime}\left(G_{i} ; \mathcal{H}_{\mid G_{i}}^{(\mathrm{t})}, \mathcal{K}_{\mid G_{i}}\right)$ as defined above. 
Elements of $\operatorname{Out}^{\prime}\left(G ; \mathcal{H}^{(\mathrm{t})}, \mathcal{K}\right)$ leave every $A_{j}$ invariant (up to conjugacy) and we denote by

$$
\theta: \operatorname{Out}^{\prime}\left(G ; \mathcal{H}^{(\mathrm{t})}, \mathcal{K}\right) \longrightarrow \prod_{j} \operatorname{Out}\left(A_{j}\right)
$$

the natural map.

We can now state:

Proposition 3.10 Let $\mathcal{H}$ and $\mathcal{K}$ be two finite families of abelian subgroups and let $\operatorname{Out}^{\prime}\left(G ; \mathcal{H}^{(\mathrm{t})}, \mathcal{K}\right)$ be the finite-index subgroup of $\operatorname{Out}\left(G ; \mathcal{H}^{(\mathrm{t})}, \mathcal{K}\right)$ defined above.

There are subgroups $F_{j} \subset A_{j}$ such that the image of $\theta$ : $\operatorname{Out}^{\prime}\left(G ; \mathcal{H}^{(\mathrm{t})}, \mathcal{K}\right) \rightarrow \prod_{j} \operatorname{Out}\left(A_{j}\right)$ equals $\prod_{j} \operatorname{Out}\left(A_{j} ;\left\{F_{j}\right\}^{(\mathrm{t})}, \mathcal{K}_{\mid A_{j}}\right)$.

Recall that the $A_{j}$ are representatives of conjugacy classes of non-cyclic maximal abelian subgroups.

Proof The $A_{j}$ are contained (up to conjugacy) in factors $G_{i}$ of the Grushko decomposition relative to $\mathcal{H} \cup \mathcal{K}$ and the $G_{i}$ are invariant under $\operatorname{Out}^{\prime}\left(G ; \mathcal{H}^{(\mathrm{t})}, \mathcal{K}\right)$. Since any family of automorphisms $\Phi_{i} \in \operatorname{Out}^{\prime}\left(G_{i} ; \mathcal{H}_{\mid G_{i}}^{(\mathrm{t})}, \mathcal{K}_{\mid G_{i}}\right)$ extends to an automorphism $\Phi \in \operatorname{Out}^{\prime}\left(G ; \mathcal{H}^{(\mathrm{t})}, \mathcal{K}\right)$, we may assume that $G$ is freely indecomposable relative to $\mathcal{H} \cup \mathcal{K}$.

Let $T_{\text {can }}$ be as above. If $A_{j}$ is contained in a rigid vertex stabilizer, then $\operatorname{Out}^{\prime}\left(G ; \mathcal{H}^{(\mathrm{t})}, \mathcal{K}\right)$ acts trivially on $A_{j}$ and we define $F_{j}=A_{j}$. If not, $A_{j}$ is a vertex stabilizer $G_{v}$. Vertex stabilizers adjacent to $v$ are rigid or $\mathrm{QH}$ and, because of the way we defined it, $\operatorname{Out}^{\prime}\left(G ; \mathcal{H}^{(\mathrm{t})}, \mathcal{K}\right)$ leaves $A_{j}$ invariant and acts trivially on incident edge groups. It also acts trivially on the groups belonging to $\mathcal{H}_{\mid A_{j}}$.

Defining $F_{j}$ as the subgroup of $A_{j}$ generated by incident edge groups and groups in $\mathcal{H}_{\mid A_{j}}$, we have proved that the image of $\theta$ is contained in $\prod_{j} \operatorname{Out}\left(A_{j} ;\left\{F_{j}\right\}^{(\mathrm{t})}, \mathcal{K}_{\mid A_{j}}\right)$. Conversely, choose a family $\Phi_{j} \in \operatorname{Out}\left(A_{j} ;\left\{F_{j}\right\}^{(\mathrm{t})}, \mathcal{K}_{\mid A_{j}}\right)$. As explained in Section 2.3, there exists $\Phi \in \mathrm{Out}^{0}\left(T_{\text {can }}\right)$ acting trivially on cyclic, rigid and QH vertex stabilizers and inducing $\Phi_{j}$ on $A_{j}$. We check that $\Phi$ acts trivially on any $H \in \mathcal{H}$. Such a group $H$ fixes a vertex $v \in T_{\text {can }}$. If $G_{v}$ is cyclic, rigid or $\mathrm{QH}$, the action of $\Phi$ on $H$ is trivial. If not, $G_{v}$ is (conjugate to) an $A_{j}$ and the action is trivial because $H \subset F_{j}$. A similar argument shows that $\Phi$ preserves $\mathcal{K}$ up to conjugacy, so $\Phi \in \operatorname{Out}\left(G ; \mathcal{H}^{(t)}, \mathcal{K}\right)$. Since $\Phi$ acts trivially on rigid and $\mathrm{QH}$ vertex stabilizers, $\Phi \in \operatorname{Out}^{\prime}\left(G ; \mathcal{H}^{(\mathrm{t})}, \mathcal{K}\right)$. 


\subsection{Automorphisms preserving a tree}

We now study the stabilizer of a tree. The following theorem clearly implies Theorem 1.4.

Theorem 3.11 Let $G$ be a toral relatively hyperbolic group. Let $T$ be a simplicial tree on which $G$ acts with abelian edge stabilizers. Let $\mathcal{K}$ be a finite family of abelian subgroups of $G$, each of which fixes a point in $T$. Then $\operatorname{Out}(T, \mathcal{K})=\operatorname{Out}(T) \cap \operatorname{Out}(G ; \mathcal{K})$ is of type $\mathrm{VF}$.

The group $\operatorname{Out}(T, \mathcal{K})$ is the subgroup of $\operatorname{Out}(G)$ consisting of automorphisms leaving $T$ invariant and mapping each group of $\mathcal{K}$ to a conjugate (in an arbitrary way). The tree $T$ is assumed to be minimal, but it may be a point, it may have trivial edge stabilizers, and non-cyclic abelian subgroups need not be elliptic.

Theorem 3.4 proves Theorem 3.11 when $T$ is a point. Also note that, if $G$ is abelian and $T$ is not a point, then $T$ is a line on which $G$ acts by integral translations and $\operatorname{Out}(T, \mathcal{K})$ is of type VF because it equals $\operatorname{Out}(G ; \mathcal{K} \cup\{N\})$, with $N$ the kernel of the action of $G$ on $T$.

Thus, we assume from now on that $G$ is not abelian. We will prove Theorem 3.11 when $T$ has cyclic edge stabilizers before treating the general case. This special case is much easier because $\operatorname{Out}\left(G_{e}\right)$ is finite for every edge stabilizer $G_{e}$ and we may apply [27, Proposition 2.3].

3.2.1 Cyclic edge stabilizers In this subsection we prove Theorem 3.11 when all edge stabilizers $G_{e}$ of $T$ are cyclic (possibly trivial); this happens in particular if $G$ is hyperbolic.

As in Section 2.3, we consider the exact sequence

$$
1 \longrightarrow \mathcal{T} \longrightarrow \operatorname{Out}^{0}(T) \stackrel{\rho}{\longrightarrow} \prod_{v \in V} \operatorname{Out}\left(G_{v} ; \operatorname{Inc}_{v}\right) .
$$

The image of $\rho$ contains $\prod_{v \in V} \operatorname{Out}\left(G_{v} ; \operatorname{Inc}_{v}^{(\mathrm{t})}\right)$ and the index is finite because all groups $\operatorname{Out}\left(G_{e}\right)$ are finite (see [27], where $\operatorname{Out}\left(G_{v} ; \operatorname{Inc}_{v}^{(\mathrm{t})}\right)$ is denoted by $\operatorname{PMCG}\left(G_{v}\right)$ ). The preimage of $\prod_{v \in V} \operatorname{Out}\left(G_{v} ; \operatorname{Inc}_{v}^{(\mathrm{t})}\right)$ is thus a finite index subgroup $\operatorname{Out}^{1}(T) \subset \operatorname{Out}(T)$.

We want to prove that $\operatorname{Out}(T, \mathcal{K})$ is of type $\mathrm{VF}$, so we restrict the preceding discussion to $\operatorname{Out}(T, \mathcal{K})$. Let

$$
\operatorname{Out}^{1}(T, \mathcal{K})=\operatorname{Out}^{1}(T) \cap \operatorname{Out}(G ; \mathcal{K}),
$$

a finite-index subgroup. We show that $\operatorname{Out}^{1}(T, \mathcal{K})$ is of type VF (this will not use the assumption that edge stabilizers are cyclic). 
The image of $\operatorname{Out}^{1}(T, \mathcal{K})$ by $\rho$ is contained in $\prod_{v \in V} \operatorname{Out}\left(G_{v} ; \operatorname{Inc}_{v}^{(\mathrm{t})}, \mathcal{K}_{\| G_{v}}\right)$, with $\mathcal{K}_{\| G_{v}}$ as in Section 2.1 and, arguing as in Section 2.3, one sees that equality holds. On the other hand, Out $^{1}(T, \mathcal{K})$ contains $\mathcal{T}$ because twists act trivially on vertex stabilizers, hence on $\mathcal{K}$ since groups of $\mathcal{K}$ are elliptic in $T$. We therefore have an exact sequence

$$
1 \longrightarrow \mathcal{T} \longrightarrow \operatorname{Out}^{1}(T, \mathcal{K}) \longrightarrow \prod_{v \in V} \operatorname{Out}\left(G_{v} ; \operatorname{Inc}_{v}^{(\mathrm{t})}, \mathcal{K}_{\| G_{v}}\right) \longrightarrow 1
$$

Vertex stabilizers are toral relatively hyperbolic, so the product is of type VF by Theorem 3.4 applied to the $G_{v}$. We conclude the proof by showing that $\mathcal{T}$ is of type $\mathrm{F}$. This will imply that $\operatorname{Out}^{1}(T, \mathcal{K})$, and hence $\operatorname{Out}(T, \mathcal{K})$, is VF.

We claim that $\mathcal{T}$ is isomorphic to the direct product of a finitely generated abelian group and a finite number of copies of non-abelian vertex groups $G_{v}$. We use the presentation of $\mathcal{T}$ given in [27, Proposition 3.1]. It says that $\mathcal{T}$ can be written as a quotient

$$
\mathcal{T}=\prod_{e, v} Z_{G_{v}}\left(G_{e}\right) /\left\langle\mathcal{R}_{V}, \mathcal{R}_{E}\right\rangle
$$

the product being taken over all pairs $(e, v)$ where $e$ is an edge incident to $v$; here $\mathcal{R}_{E}=\prod_{e} Z\left(G_{e}\right)$ is the group of edge relations and $\mathcal{R}_{V}=\prod_{v} Z\left(G_{v}\right)$ is the group of vertex relations, both embedded naturally in $\prod_{e, v} Z_{G_{v}}\left(G_{e}\right)$. Every group $Z_{G_{v}}\left(G_{e}\right)$ is abelian, unless $G_{e}$ is trivial and $G_{v}$ is non-abelian. In this case $Z_{G_{v}}\left(G_{e}\right)=G_{v}$ and it is not affected by the edge and vertex relations since both $Z\left(G_{v}\right)$ and $Z\left(G_{e}\right)$ are trivial. Our claim follows.

It follows that $\mathcal{T}$ is of type $\mathrm{F}$ provided that it is torsion-free. One may show that this is always the case, but it is simpler to replace $\operatorname{Out}^{1}(T, \mathcal{K})$ by its intersection with a torsion-free, finite-index subgroup of $\operatorname{Out}(G)$.

\subsubsection{Changing $\boldsymbol{T}$ We shall now prove Theorem 3.11 in the general case.}

The first step, carried out in this subsection, is to replace $T$ by a better tree $\widehat{T}$ (satisfying the second assertion of the lemma below). When all edge stabilizers are non-trivial, $\widehat{T}$ may be viewed as the smallest common refinement (called lcm in [22]) of $T$ and its tree of cylinders (see Section 2.2). Here is the construction of $\widehat{T}$.

Consider edges of $T$ with non-trivial stabilizer. We say that two such edges belong to the same cylinder if their stabilizers commute. Cylinders are subtrees and meet in at most one point. A vertex $v$ with all incident edge groups trivial belongs to no cylinder. Otherwise $v$ belongs to one cylinder if $G_{v}$ is abelian and to infinitely many cylinders if $G_{v}$ is not abelian. To define $\widehat{T}$, we shall refine $T$ at vertices $x$ belonging to infinitely many cylinders. 
Given such an $x$, let $S_{x}$ be the set of cylinders $Y$ such that $x \in Y$. We replace $x$ by the cone $T_{x}$ on $S_{x}$ : there is a central vertex, again denoted by $x$, and vertices $\left(x, s_{Y}\right)$ for $Y \in S_{x}$, with an edge between $x$ and $\left(x, s_{Y}\right)$. Edges $e$ of $T$ incident to $x$ are attached to $T_{x}$ as follows: if the stabilizer of $e$ is trivial, we attach it to the central vertex $x$; if not, $e$ is contained in a cylinder $Y$ and we attach $e$ to the vertex $\left(x, s_{Y}\right)$, noting that $G_{e}$ leaves $Y$ invariant.

Performing this operation at each $x$ belonging to infinitely many cylinders yields a tree $\widehat{T}$. The construction being canonical, there is a natural action of $G$ on $\widehat{T}$, and $\operatorname{Out}(T) \subset \operatorname{Out}(\widehat{T})$.

Lemma 3.12 (1) Edge stabilizers of $\widehat{T}$ are abelian, $\widehat{T}$ is dominated by $T$, and $\operatorname{Out}(\widehat{T})=\operatorname{Out}(T)$.

(2) Let $G_{v}$ be a non-abelian vertex stabilizer of $\widehat{T}$. Non-trivial incident edge stabilizers $G_{e}$ are maximal abelian subgroups of $G_{v}$. If $e_{1}$ and $e_{2}$ are edges of $\widehat{T}$ incident to $v$ with $G_{e_{1}}$ and $G_{e_{2}}$ equal and non-trivial, then $e_{1}=e_{2}$.

Proof Let $Y$ be a cylinder in $S_{x}$ (viewed as a subtree of $T$ ). The setwise stabilizer $G_{Y}$ of $Y$ is the maximal abelian subgroup of $G$ containing stabilizers of edges of $Y$. The stabilizer of the vertex $\left(x, s_{Y}\right)$ of $\widehat{T}$, and also of the edge between $\left(x, s_{Y}\right)$ and $x$, is $G_{X} \cap G_{Y}$; it is non-trivial (it contains the stabilizer of edges of $Y$ incident to $x$ ) and is a maximal abelian subgroup of $G_{x}$. This proves that edge stabilizers of $\widehat{T}$ are abelian, since the other edges have the same stabilizer as in $T$.

Every vertex stabilizer of $T$ is also a vertex stabilizer of $\widehat{T}$, so $T$ dominates $\widehat{T}$. Edges of $\widehat{T}$ which are not edges of $T$ (those between $\left(x, s_{Y}\right)$ and $x$ ) are characterized as those having non-trivial stabilizer and having an endpoint $v$ with $G_{v}$ non-abelian. One recovers $T$ from $\widehat{T}$ by collapsing these edges, so $\operatorname{Out}(\widehat{T}) \subset \operatorname{Out}(T)$.

Consider two edges $e_{1}$ and $e_{2}$ incident to $v$ in $\widehat{T}$, with the same non-trivial stabilizer. They join $v$ to vertices $\left(v, s_{Y_{i}}\right)$ and we have seen that $G_{e_{1}}=G_{e_{2}}$ is maximal abelian in $G_{v}$. The groups $G_{Y_{1}}$ and $G_{Y_{2}}$ are equal because they both contain $G_{e_{1}}=G_{e_{2}}$. Edges of $Y_{i}$ have stabilizers contained in $G_{Y_{i}}$, so have commuting stabilizers. Thus $Y_{1}=Y_{2}$, so $e_{1}=e_{2}$.

Remark 3.13 If $G_{e_{1}}$ and $G_{e_{2}}$ are conjugate in $G_{v}$, rather than equal, we conclude that $e_{1}$ and $e_{2}$ belong to the same $G_{v}$-orbit. On the other hand, edges belonging to different $G_{v}$-orbits may have stabilizers which are conjugate in $G$ (but not in $G_{v}$ ). 
3.2.3 The action on edge groups In Section 3.2.1 we could neglect the action of $\operatorname{Out}^{0}(T)$ on edge groups because all groups $\operatorname{Out}\left(G_{e}\right)$ were finite. We now allow edge stabilizers of arbitrary rank, so we must take these actions into account. We write $\operatorname{Out}^{0}(T, \mathcal{K})=\operatorname{Out}^{0}(T) \cap \operatorname{Out}(G ; \mathcal{K})$.

Recall that, for each edge $e$ of $\Gamma=T / G$, there is a natural map $\rho_{e}: \operatorname{Out}^{0}(T) \rightarrow \operatorname{Out}\left(G_{e}\right)$ (see Section 2.3). The collection of all these maps defines a map

$$
\psi: \operatorname{Out}^{0}(T, \mathcal{K}) \longrightarrow \prod_{e \in E} \operatorname{Out}\left(G_{e}\right),
$$

the product being over all non-oriented edges of $\Gamma$. We denote by $Q$ the image of $\operatorname{Out}^{0}(T, \mathcal{K})$ under $\psi$, so that we have the exact sequence

$$
1 \longrightarrow \operatorname{ker} \psi \longrightarrow \operatorname{Out}^{0}(T, \mathcal{K}) \longrightarrow Q \longrightarrow 1 \text {. }
$$

Lemma 3.14 If $T$ satisfies the second assertion of Lemma 3.12, then the group $Q$ is of type VF.

This lemma will be proved in the next subsection. We first explain how to deduce Theorem 3.11 from it. The first assertion of Lemma 3.12 implies that the theorem holds for $T$ if it holds for $\widehat{T}$, so we may assume that $T$ satisfies the second assertion of Lemma 3.12.

The kernel of $\psi$ is the group discussed in Section 3.2.1 under the name $\operatorname{Out}^{1}(T, \mathcal{K})$, but now (contrary to Convention 3.5) $\operatorname{Out}^{1}(T, \mathcal{K})$ may be of infinite index $\operatorname{in} \operatorname{Out}(T, \mathcal{K})$; indeed, $\operatorname{Out}(T, \mathcal{K})$ is virtually an extension of $\operatorname{Out}^{1}(T, \mathcal{K})$ by $Q$. To avoid confusion, we use the notation ker $\psi$ rather than $\operatorname{Out}^{1}(T, \mathcal{K})$.

We proved in Section 3.2.1 that ker $\psi$ is of type VF and, by the lemma, $Q$ is of type VF, but this is not quite sufficient (see Remark 3.3). We shall now construct a finite-index subgroup $\operatorname{Out}^{2}(T, \mathcal{K}) \subset \operatorname{Out}^{0}(T, \mathcal{K})$ such that ker $\psi \cap \operatorname{Out}^{2}(T, \mathcal{K})$ has type $\mathrm{F}$. Applying Corollary 3.2 to $\operatorname{Out}^{0}(T, \mathcal{K})$ then completes the proof of Theorem 3.11.

We argue as in Section 3.2.1. Recall from Section 2.3 the exact sequence

$$
1 \longrightarrow \mathcal{T} \longrightarrow \operatorname{Out}^{0}(T, \mathcal{K}) \stackrel{\rho}{\longrightarrow} \prod_{v \in V} \operatorname{Out}\left(G_{v} ; \operatorname{Inc}_{v}, \mathcal{K}_{\| G_{v}}\right)
$$

whose restriction to ker $\psi$ is the exact sequence

$$
1 \longrightarrow \mathcal{T} \longrightarrow \operatorname{ker} \psi \stackrel{\rho}{\longrightarrow} \prod_{v \in V} \operatorname{Out}\left(G_{v} ; \operatorname{Inc}_{v}^{(\mathrm{t})}, \mathcal{K}_{\| G_{v}}\right) \longrightarrow 1
$$


Using the "more precise" statement of Theorem 3.4 we get, for each $v \in V$, a finite-index subgroup $\operatorname{Out}^{1}\left(G_{v} ; \operatorname{Inc}_{v}, \mathcal{K}_{\| G_{v}}\right) \subset \operatorname{Out}\left(G_{v} ; \operatorname{Inc}_{v}, \mathcal{K}_{\| G_{v}}\right)$ such that

$$
\operatorname{Out}^{1}\left(G_{v} ; \operatorname{Inc}_{v}, \mathcal{K}_{\| G_{v}}\right) \cap \operatorname{Out}\left(G_{v} ; \operatorname{Inc}_{v}^{(\mathrm{t})}, \mathcal{K}_{\| G_{v}}\right)
$$

is of type F. Define the finite-index subgroup $\operatorname{Out}^{2}(T, \mathcal{K}) \subset \operatorname{Out}^{0}(T, \mathcal{K})$ as the preimage of $\prod_{v \in V} \operatorname{Out}^{1}\left(G_{v} ; \operatorname{Inc}_{v}, \mathcal{K}_{\| G_{v}}\right)$ under $\rho$ intersected with a torsion-free, finite-index subgroup of $\operatorname{Out}(G)$.

Restricting the exact sequence above, we get an exact sequence

$$
1 \longrightarrow \mathcal{T}^{\prime} \longrightarrow \operatorname{ker} \psi \cap \operatorname{Out}^{2}(T, \mathcal{K}) \stackrel{\rho}{\longrightarrow} L \longrightarrow 1,
$$

where $L$ has finite index in the product of the groups

$$
\operatorname{Out}^{1}\left(G_{v} ; \operatorname{Inc}_{v}, \mathcal{K}_{\| G_{v}}\right) \cap \operatorname{Out}\left(G_{v} ; \operatorname{Inc}_{v}^{(\mathrm{t})}, \mathcal{K}_{\| G_{v}}\right),
$$

hence has type $\mathrm{F}$. The group $\mathcal{T}^{\prime}$ is a torsion-free, finite-index subgroup of $\mathcal{T}$, so has type $\mathrm{F}$ as in Section 3.2.1. We conclude that $\operatorname{ker} \psi \cap \operatorname{Out}^{2}(T, \mathcal{K})$ has type $\mathrm{F}$. As explained above, this completes the proof of Theorem 3.11 (assuming Lemma 3.14).

3.2.4 Proof of Lemma 3.14 There remains to prove Lemma 3.14. We let $E_{j}$ be representatives of conjugacy classes of maximal abelian subgroups containing a nontrivial edge stabilizer. Note that $E_{j}$ is allowed to be cyclic and maximal abelian subgroups of $G$ containing no non-trivial $G_{e}$ are not included.

Inside each $E_{j}$ we let $B_{j}$ be the smallest direct factor containing all edge groups included in $E_{j}$ (it equals $E_{j}$ if $E_{j}$ is cyclic). It is elliptic in $T$, because it is an abelian group generated (virtually) by elliptic subgroups.

Each automorphism $\Phi \in \operatorname{Out}^{0}(T, \mathcal{K})$ induces an automorphism of $E_{j}$, which preserves $B_{j}$ and all the edge groups it contains. This defines a map

$$
\psi^{\prime}: \operatorname{Out}^{0}(T, \mathcal{K}) \longrightarrow \prod_{j} \operatorname{Out}\left(B_{j}\right)
$$

having the same kernel as the map $\psi: \operatorname{Out}^{0}(T, \mathcal{K}) \rightarrow \prod_{e \in E} \operatorname{Out}\left(G_{e}\right)$ defined in Section 3.2.3. Thus, it suffices to prove that the image of $\operatorname{Out}^{0}(T, \mathcal{K})$ by $\psi^{\prime}$ is of type VF. We do so by finding a finite-index subgroup $\operatorname{Out}^{1}(T, \mathcal{K})$ (not the same as in Section 3.2.1) whose image is a product $\prod_{j} Q_{j}$ with each $Q_{j}$ of type VF.

Consider a non-abelian vertex group $G_{v}$. Define $\operatorname{Inc}_{v, \mathbb{Z}} \subset \operatorname{Inc}_{v}$ by keeping only the incident edge groups which are infinite cyclic, and denote by $E_{\mathrm{nc}}(v)$ the set of edges $e$ of $\Gamma$ with origin $v$ and $G_{e}$ non-cyclic (if $e$ is a loop, we subdivide it so that it 
counts twice in $\left.E_{\mathrm{nc}}(v)\right)$. By Lemma 3.12 and Remark 3.13, the edge groups $G_{e}$ for $e \in E_{\mathrm{nc}}(v)$ are non-conjugate maximal abelian subgroups of $G_{v}$.

We apply Proposition 3.10, describing the action on non-cyclic maximal abelian subgroups, to $\operatorname{Out}\left(G_{v} ; \operatorname{Inc}_{v, \mathbb{Z}}^{(\mathrm{t})}, \mathcal{K}_{\| G_{v}}\right)$. We get a subgroup $\operatorname{Out}^{\prime}\left(G_{v} ; \operatorname{Inc}_{v, \mathbb{Z}}^{(\mathrm{t})}, \mathcal{K}_{\| G_{v}}\right)$ of finite index and a subgroup $F_{e}^{v} \subset G_{e}$ for each edge $e \in E_{\mathrm{nc}}(v)$ such that the image of $\operatorname{Out}^{\prime}\left(G_{v} ; \operatorname{Inc}_{v, \mathbb{Z}}^{(\mathrm{t})}, \mathcal{K}_{\| G_{v}}\right)$ in $\prod_{e \in E_{\mathrm{nc}}(v)} \operatorname{Out}\left(G_{e}\right)$ is $\prod_{e \in E_{\mathrm{nc}}(v)} \operatorname{Out}\left(G_{e} ;\left\{F_{e}^{v}\right\}^{(\mathrm{t})}, \mathcal{K}_{\mid G_{e}}\right)$.

We let $\operatorname{Out}^{1}(T, \mathcal{K}) \subset \operatorname{Out}^{0}(T, \mathcal{K})$ be the subgroup consisting of automorphisms acting trivially on cyclic edge stabilizers and acting on non-abelian vertex stabilizers as an element of $\operatorname{Out}^{\prime}\left(G_{v} ; \operatorname{Inc}_{v, \mathbb{Z}}^{(\mathrm{t})}, \mathcal{K}_{\| G_{v}}\right)$. It has finite index because

$$
\operatorname{Out}\left(G_{v} ; \operatorname{Inc}_{v, \mathbb{Z}}^{(\mathrm{t})}, \mathcal{K}_{\| G_{v}}\right) \subset \rho_{v}\left(\operatorname{Out}^{0}(T, \mathcal{K})\right) \subset \operatorname{Out}\left(G_{v} ; \operatorname{Inc}_{v, \mathbb{Z}}, \mathcal{K}_{\| G_{v}}\right),
$$

with all indices finite.

We now define $Q_{j} \subset \operatorname{Out}\left(B_{j}\right)$ as consisting of automorphisms $\Phi_{j}$ such that

(1) if $G_{e}$ is a cyclic edge stabilizer contained in $B_{j}$, then $\Phi_{j}$ acts trivially on $G_{e}$;

(2) if $B_{j}$ contains a non-cyclic $G_{e}$ and $v$ is an endpoint of $e$ with $G_{v}$ non-abelian, then $\Phi_{j}$ acts trivially on $F_{e}^{v}$;

(3) non-cyclic edge stabilizers and abelian vertex stabilizers contained in $B_{j}$ are $\Phi_{j}$-invariant;

(4) $\Phi_{j}$ extends to an automorphism of $E_{j}$ leaving $\mathcal{K}_{\mid E_{j}}$ invariant; in particular, subgroups of $B_{j}$ conjugate to a group of $\mathcal{K}$ are $\Phi_{j}$-invariant.

This definition was designed so that the image of $\operatorname{Out}^{1}(T, \mathcal{K})$ by $\psi^{\prime}$ is contained in $\prod_{j} Q_{j}$. We claim that equality holds:

Lemma 3.15 The image of $\operatorname{Out}^{1}(T, \mathcal{K})$ by $\psi^{\prime}$ equals $\prod_{j} Q_{j}$.

Proof We fix automorphisms $\Phi_{j} \in Q_{j} \subset \operatorname{Out}\left(B_{j}\right)$ and we have to construct an automorphism $\Phi \in \operatorname{Out}^{1}(T, \mathcal{K})$. By (1) and (3) above, the $\Phi_{j}$ induce automorphisms $\Phi_{e}$ of edge stabilizers (each non-trivial edge group $G_{e}$ lies in a unique $E_{j}$, so there is no ambiguity in the definition of $\Phi_{e}$ ). As explained after Lemma 2.2, it suffices to find automorphisms $\Phi_{v}$ of vertex groups inducing the $\Phi_{e}$. We distinguish several cases.

If $G_{v}$ is contained in some $B_{j}$ (up to conjugacy), it is $\Phi_{j}$-invariant by (3), so we let $\Phi_{v}$ be the restriction.

If $G_{v}$ is abelian but not contained in any $B_{j}$, we may assume that some incident $G_{e}$ is non-cyclic (otherwise we let $\Phi_{v}$ be the identity). This $G_{e}$ is contained in some $B_{j}$, 
and $G_{v} \subset E_{j}$. In fact, $G_{v}=E_{j}$ : since $G_{v}$ is not contained in $B_{j}$, it fixes only $v$, and $E_{j}$ fixes $v$ because it commutes with $G_{v}$. We may thus extend $\Phi_{j}$ to $G_{v}$ using (4). If $G_{v}$ is not abelian, we construct $\Phi_{v}$ in $\operatorname{Out}^{\prime}\left(G_{v} ; \operatorname{Inc}_{v, \mathbb{Z}}^{(\mathrm{t})}, \mathcal{K}_{\| G_{v}}\right)$ as follows. If $e \in E_{\mathrm{nc}}(v)$, the automorphism $\Phi_{e}$ acts trivially on $F_{e}^{v}$ by (2), and preserves $\mathcal{K}_{\mid G_{e}}$ by (4). Thus, the collection of automorphisms $\Phi_{e}$ lies in $\prod_{e \in E_{\mathrm{nc}}(v)} \operatorname{Out}\left(G_{e} ;\left\{F_{e}^{v}\right\}^{(t)}, \mathcal{K}_{\mid G_{e}}\right)$. Proposition 3.10 guarantees that $\operatorname{Out}^{\prime}\left(G_{v} ; \operatorname{Inc}_{v, \mathbb{Z}}^{(\mathrm{t})}, \mathcal{K}_{\| G_{v}}\right)$ contains an automorphism $\Phi_{v}$ inducing $\Phi_{e}$ for all $e \in E_{\mathrm{nc}}(v)$ (and acting trivially on all cyclic incident edge groups).

We have now constructed automorphisms $\Phi_{v} \in \operatorname{Out}\left(G_{v}\right)$ inducing the $\Phi_{e}$, so Lemma 2.2 provides an automorphism $\Phi \in \operatorname{Out}^{0}(T)$ whose image in $\prod_{j} \operatorname{Out}\left(B_{j}\right)$ is the product of the $\Phi_{j}$ because $B_{j}$ is virtually generated by edge stabilizers. We show $\Phi \in \operatorname{Out}^{1}(T, \mathcal{K})$. By construction it acts trivially on cyclic edge groups and acts on non-abelian vertex stabilizers as an element of $\operatorname{Out}^{\prime}\left(G_{v} ; \operatorname{Inc}_{v, \mathbb{Z}}^{(\mathrm{t})}, \mathcal{K}_{\| G_{v}}\right)$. We just have to check that $\Phi$ leaves any $K \in \mathcal{K}$ invariant.

The group $K$ is contained in some $G_{v}$. If $K$ is contained in some $B_{j}$, it is $\Phi$-invariant by (4). Otherwise, $K$ fixes no edge. If $G_{v}$ is abelian, we have seen that either all incident edge groups are cyclic (and $\Phi_{v}$ is the identity) or $G_{v}$ equals some $E_{j}$ and our choice of $\Phi_{v}$ using (4) guarantees that $K$ is invariant. If $G_{v}$ is not abelian, then $K$ belongs to $\mathcal{K}_{\| G_{v}}$ because it fixes no edge. It is invariant because we chose $\Phi_{v} \in \operatorname{Out}^{\prime}\left(G_{v} ; \operatorname{Inc}_{v, \mathbb{Z}}^{(\mathrm{t})}, \mathcal{K}_{\| G_{v}}\right)$.

We have seen that the group $Q$ of Lemma 3.14 is isomorphic to the image of $\operatorname{Out}^{0}(T, \mathcal{K})$ under $\psi^{\prime}$, hence contains $\prod_{j} Q_{j}$ with finite index. To show that $Q$ is of type VF, there remains to show that each $Q_{j}$ is of type VF.

We defined $Q_{j}$ inside $\operatorname{Out}\left(B_{j}\right)$ by four conditions. As in Lemma 3.6, the first three define an arithmetic group. To deal with the fourth one, we consider the group $\widetilde{Q}_{j}$ consisting of automorphisms of $E_{j}$ that leave $B_{j}$ and $\mathcal{K}_{\mid E_{j}}$ invariant with the restriction to $B_{j}$ satisfying the first three conditions. This is an arithmetic group. It consists of block-triangular matrices and one obtains $Q_{j}$ by considering the upper-left blocks of matrices in $\widetilde{Q}_{j}$. It follows that $K_{j}$ is arithmetic, as the image of an arithmetic group by a rational homomorphism [6, Theorem 6], hence of type VF by Lemma 3.6.

This completes the proof of Lemma 3.14, and hence of Theorem 3.11.

\section{A finiteness result for trees}

The goal of this subsection is Proposition 4.8, which gives a uniform bound for the size of certain sets of relative JSJ decompositions of $G$. This an essential ingredient in the 
proof of the chain condition for McCool groups. We will have to restrict to root-closed (RC) trees, which are introduced in Definitions 4.3 and 4.7 (they are closely related to the primary splittings of Dahmani and Groves [11]).

Definition 4.1 Let $H$ be a subgroup of a group $G$. Its root closure $e(H, G)$, or simply $e(H)$, is the set of elements of $G$ having a power in $H$. If $e(H)=H$, we say that $H$ is root-closed.

If $G$ is toral relatively hyperbolic and $H$ is abelian, $e(H)$ is a direct factor of the maximal abelian subgroup containing $H$, and $H$ has finite index in $e(H)$. Also note that, given $h \in G$ and $n \geq 2$, there exists at most one element $g$ such that $g^{n}=h$.

The following fact is completely general:

Lemma 4.2 Let $T$ be a tree with an action of an arbitrary group. The following are equivalent:

- Vertex stabilizers of $T$ are root-closed.

- Edge stabilizers of $T$ are root-closed.

Proof If $g^{n}$ fixes an edge $e=v w$, it fixes $v$ and $w$. If vertex stabilizers are root-closed, $g$ fixes $v$ and $w$, hence fixes $e$, so edge stabilizers are root-closed.

Conversely, if $g^{n}$ fixes a vertex $v$, then $g$ is elliptic, hence fixes a vertex $w$. Edges between $v$ and $w$ (if any) are fixed by $g^{n}$, hence by $g$ if edge stabilizers are root-closed. Thus $g$ fixes $v$.

We now go back to a toral relatively hyperbolic group $G$.

Definition 4.3 A tree $T$ is an $R C$ tree if

- all non-cyclic abelian subgroups fix a point in $T$;

- edge stabilizers of $T$ are abelian and root-closed.

When $G$ is hyperbolic, RC trees are the $\mathcal{Z}_{\max }$-trees of Dahmani and Guirardel [12]: non-trivial edge stabilizers are maximal cyclic subgroups.

Lemma 4.4 (1) Let $T$ be an $R C$ tree with all edge stabilizers non-trivial. Its tree of cylinders $T_{c}$ (see Section 2.2) is an RC tree belonging to the same deformation space as $T$. 
(2) If $T_{1}$ and $T_{2}$ are $R C$ trees relative to some family $\mathcal{H}$ and edge stabilizers of $T_{1}$ are elliptic in $T_{2}$, there is an $R C$ tree $\widehat{T}_{1}$ relative to $\mathcal{H}$ which refines $T_{1}$ and dominates $T_{2}$. Moreover, the stabilizer of any edge of $\widehat{T}_{1}$ fixes an edge in $T_{1}$ or in $T_{2}$.

Proof Non-triviality of edge stabilizers ensures that $T_{c}$ is defined. The vertex stabilizers of $T_{c}$ are vertex stabilizers of $T$ or maximal abelian subgroups, so are root-closed. The deformation space does not change because $T$ is relative to non-cyclic abelian subgroups (see [23, Proposition 6.3]). This proves (1).

We define a refinement $\widehat{T}_{1}$ of $T_{1}$ dominating $T_{2}$ as in [21, Lemma 3.2], by blowing up each vertex $v$ of $T_{1}$ into a $G_{v}$-invariant subtree of $T_{2}$. We just have to check that its edge stabilizers are root-closed. As in the proof of [12, Lemma 4.9], an edge stabilizer of $\widehat{T}_{1}$ is an edge stabilizer of $T_{1}$ or is the intersection of a vertex stabilizer of $T_{1}$ with an edge stabilizer of $T_{2}$, so is root-closed.

Proposition 4.5 Let $G$ be toral relatively hyperbolic. In each of the following two cases, there is a bound for the number of orbits of edges of a minimal tree $T$ with abelian edge stabilizers:

(1) $T$ is bipartite: each edge has exactly one endpoint with abelian stabilizer (redundant vertices are allowed).

(2) $T$ is an $R C$ tree with no redundant vertex.

Here and below, the bound has to depend only on $G$ (it is independent of the trees under consideration).

Case 1 applies in particular to trees of cylinders.

Proof We cannot apply Bestvina and Feighn's accessibility theorem [3] directly because $T$ does not have to be reduced in the sense of [3]: $\Gamma=T / G$ may have a vertex $v$ of valence 2 such that an incident edge carries the same group as $v$. We say that such a $v$ is a non-reduced vertex. The assumptions rule out the possibility that $\Gamma$ contains long segments consisting of non-reduced vertices (as in the example at the top of [3, page 450]).

If $T$ is bipartite, consider all non-reduced vertices of $\Gamma$ and collapse exactly one of the incident edges. This yields a reduced graph of groups, and at most half of the edges of $\Gamma$ are collapsed, so [3] gives a bound. 
If $T$ is an RC tree with no redundant vertex, every non-reduced vertex $v$ of $\Gamma=T / G$ has exactly two adjacent edges $e_{v}$ and $f_{v}$, whose groups satisfy $G_{e_{v}} \varsubsetneqq G_{v}=G_{f_{v}}$. Among all edges incident to a non-reduced vertex, consider the set $E_{m}$ consisting of those with $G_{e}$ of minimal rank. No two edges of $E_{m}$ are adjacent at a non-reduced vertex, because $T$ is an RC tree. Now collapse the edges in $E_{m}$.

If $I=e_{1} \cup e_{2} \cup \cdots \cup e_{k}$ is a maximal segment in the complement of the set of vertices of $\Gamma$ having degree 3 or carrying a non-abelian group, we never collapse adjacent edges $e_{i}$ and $e_{i+1}$ (and we do not collapse $e_{1}$ if $k=1$; we may collapse $e_{1}$ and $e_{3}$ if $k=3$ ). It follows that at least one third of the edges of $\Gamma$ remain after the collapse.

Repeat the process. Denote by $M$ the maximal rank of abelian subgroups of $G$. After at most $M$ steps one obtains a graph of groups which is reduced in the sense of [3], hence has at most $N$ edges for some fixed $N$. The number of edges of $\Gamma$ is bounded by $3^{M} N$.

Proposition 4.6 Given a toral relatively hyperbolic group $G$, there exists a number $M$ such that, if $T_{1} \rightarrow T_{2} \rightarrow \cdots \rightarrow T_{p}$ is a sequence of maps between $R C$ trees belonging to distinct deformation spaces, then $p \leq M$.

\section{Proof There are two steps:}

- The first step is to reduce to the case when no edge stabilizer is trivial. Consider the tree $\bar{T}_{i}$ (possibly a point) obtained from $T_{i}$ by collapsing all edges with non-trivial stabilizer. A map $T_{i} \rightarrow T_{i+1}$ cannot send an arc with non-trivial stabilizer to the interior of an edge with trivial stabilizer, so $\bar{T}_{i}$ dominates $\bar{T}_{i+1}$. Vertex stabilizers of $\bar{T}_{i}$ are free factors; there are finitely many possibilities for their isomorphism type.

Using Scott's complexity, it is shown in [16, Section 2.2] that the number of times that the deformation space $\mathcal{D}_{i}$ of $\bar{T}_{i}$ differs from that of $\bar{T}_{i+1}$ is uniformly bounded. We may therefore assume that $\mathcal{D}=\mathcal{D}_{i}$ is independent of $i$.

Let $H_{1}, \ldots, H_{k}$ be representatives of conjugacy classes of non-trivial vertex stabilizers of trees in $\mathcal{D}$. They are free factors of $G$, hence toral relatively hyperbolic, and $k$ is bounded.

Consider the action of $H_{j}$ on its minimal subtree $T_{i}^{j} \subset T_{i}$ (we let $T_{i}^{j}$ be any fixed point if the action is trivial). It is an RC tree and no edge stabilizer is trivial. The deformation space of $T_{i}$ is completely determined by $\mathcal{D}$ and the deformation spaces $\mathcal{D}_{i}^{j}$ of the trees $T_{i}^{j}$ (viewed as trees with an action of $H_{j}$ ). It therefore suffices to bound (by a constant depending only on $H_{j}$ ) the number of times that $\mathcal{D}_{i}^{j}$ changes in a sequence $T_{1}^{j} \rightarrow T_{2}^{j} \rightarrow \cdots \rightarrow T_{p}^{j}$, so we may continue the proof under the additional assumption that the $T_{i}$ have non-trivial edge stabilizers. 
- Now that edge stabilizers are non-trivial, the tree of cylinders of $T_{i}$ is defined. By the first assertion of Lemma 4.4, we may assume that it equals $T_{i}$.

Since all trees are trees of cylinders, we may assume, by [23, Proposition 4.11], that all domination maps $T_{i} \rightarrow T_{i+1}$ send vertex to vertex and map an edge to either a point or an edge. Such a map may collapse an edge to a point, or identify edges belonging to different orbits, or identify edges in the same orbit. The first two phenomena are easy to control, since they decrease the number of orbits of edges; controlling the third one requires more care (and restricting to $\mathrm{RC}$ trees).

We associate a complexity $(n,-s)$ to each $T_{i}$, with $n$ the number of edges of $T_{i} / G$ and $s$ the sum of the ranks of its edge groups; complexities are ordered lexicographically. We claim that the complexity of $T_{i+1}$ is strictly smaller than that of $T_{i}$. This gives the required uniform bound on $p$, since $n$ (hence also $s$ ) is bounded by the first case of Proposition 4.5.

Let $f_{i}: T_{i} \rightarrow T_{i+1}$ be a domination map as above. Complexity clearly cannot increase when passing from $T_{i}$ to $T_{i+1}$. If $n$ does not decrease, no edge of $T_{i}$ is collapsed in $T_{i+1}$. Since $T_{i}$ and $T_{i+1}$ belong to distinct deformation spaces, there exist distinct edges $e$ and $e^{\prime}$ identified by $f_{i}$. They have to belong to the same orbit (otherwise $n$ decreases), so $e^{\prime}=g e$ for some $g \in G$. The group $\left\langle g, G_{e}\right\rangle$ fixes the edge $f_{i}(e)=f_{i}\left(e^{\prime}\right)$ of $T_{i+1}$, so is abelian. It has rank bigger than the rank of $G_{e}$ because $G_{e}$ is root-closed and $g \notin G_{e}$. Thus $s$ increases, and the complexity decreases.

Let $\mathcal{A}$ be the family of all abelian subgroups. Let $\mathcal{H}$ be a family of subgroups of $G$. A JSJ tree (over $\mathcal{A}$ ) relative to $\mathcal{H}$ may be defined as a tree $T$ such that $T$ is relative to $\mathcal{H}$, edge stabilizers of $T$ are elliptic in every tree which is relative to $\mathcal{H}$, and $T$ dominates every tree satisfying the previous conditions (all trees are assumed to have abelian edge stabilizers). This motivates the following definition, where we require that $T$ be an RC tree (compare [12, Section 4.4]). Recall that $\mathcal{H}^{+\mathrm{ab}}$ is obtained by adding all non-cyclic abelian subgroups to $\mathcal{H}$.

Definition 4.7 Let $G$ be a toral relatively hyperbolic group and $\mathcal{H}$ a family of subgroups. A tree $T$ is an $R C J S J$ tree relative to $\mathcal{H}^{+ \text {ab }}$ if

(1) $T$ is relative to $\mathcal{H}^{+\mathrm{ab}}$ and is an RC tree;

(2) edge stabilizers of $T$ are elliptic in every (not necessarily RC) tree with abelian edge stabilizers which is relative to $\mathcal{H}^{+ \text {ab }}$;

(3) $T$ dominates every tree satisfying (1) and (2).

We will construct RC JSJ trees in Section 5. Note that non-cyclic edge stabilizers always satisfy (2). 
Proposition 4.8 Let $G$ be a toral relatively hyperbolic group. Let $\mathcal{H}_{1} \subset \cdots \subset \mathcal{H}_{i} \subset \cdots$ be an increasing sequence (finite or infinite) of families of subgroups with $G$ freely indecomposable relative to $\mathcal{H}_{1}$. For each $i$, let $U_{i}$ be an $R C J S J$ tree relative to $\mathcal{H}_{i}^{+ \text {ab }}$. There exists a number $q$, depending only on $G$, such that the trees $U_{i}$ belong to at most $q$ distinct deformation spaces.

Proof Let $U_{i}$ be as in the proposition. Note that $U_{i}$ satisfies condition (1) of Definition 4.7 with respect to $\mathcal{H}_{j}^{+\mathrm{ab}}$ if $j \leq i$ and condition (2) with respect to $\mathcal{H}_{j}^{+\mathrm{ab}}$ if $j \geq i$. But cyclic edge stabilizers of $U_{i}$ do not necessarily satisfy (2) with respect to $\mathcal{H}_{j}^{+\mathrm{ab}}$ if $j<i$.

In general, there is no domination map $U_{i} \rightarrow U_{i+1}$, so we cannot apply Proposition 4.6 directly. The easy case is when, for each $i$, every cyclic edge stabilizer of $U_{i+1}$ is contained in an edge stabilizer of $U_{i}$. Indeed, this implies that $U_{i+1}$ satisfies condition (2) with respect to $\mathcal{H}_{i}^{+\mathrm{ab}}$ (not just to $\mathcal{H}_{i+1}^{+\mathrm{ab}}$ ). By condition (3), $U_{i}$ dominates $U_{i+1}$, so Proposition 4.6 applies.

Next, assume that there is an $\mathrm{RC}$ tree $T$ relative to $\mathcal{H}_{1}$ such that, for all $i$, there is a domination map $T \rightarrow U_{i}$ that collapses no edge. Each cyclic edge stabilizer $G_{e}$ of $U_{i+1}$ contains an edge stabilizer $G_{e^{\prime}}$ of $T$ (take for $e^{\prime}$ any edge whose image contains a subarc of $e$ ). Since $G$ is freely indecomposable relative to $\mathcal{H}_{1}$ and $T$ is relative to $\mathcal{H}_{1}$, one has $G_{e^{\prime}} \neq 1$, and $G_{e^{\prime}}=G_{e}$ because $G_{e^{\prime}}$ is root-closed. Since the map $T \rightarrow U_{i}$ collapses no edge, $G_{e}$ fixes an edge in $U_{i}$ and we conclude as above.

We now construct such a tree $T$. By condition (2) of Definition 4.7, edge stabilizers of $U_{1}$ are elliptic in $U_{2}$, so by Lemma 4.4 there is an RC tree $T_{1}$ relative to $\mathcal{H}_{1}$ which refines $U_{1}$ and dominates $U_{2}$; we remove redundant vertices of $T_{1}$ if needed. Edge stabilizers of $T_{1}$ fix an edge in $U_{1}$ or $U_{2}$, so are elliptic in $U_{3}$ and one may iterate. One obtains RC trees $T_{i}$ relative to $\mathcal{H}_{1}$ such that $T_{i}$ refines $T_{i-1}$ and dominates $U_{i+1}$. By Proposition 4.5, all trees $T_{i}$ for $i$ large enough are equal to a fixed RC tree $T$. We have no control over how large $i$ has to be, but we have a uniform bound for the number of orbits of edges of $T$.

By construction, there are domination maps $f_{i}: T \rightarrow U_{i}$, but $f_{i}$ may collapse some $G$-invariant set of edges. There are only a bounded number of possibilities for the set $E_{i}$ of edges of $T$ that are collapsed by $f_{i}$, so we may assume that $E=E_{i}$ is independent of $i$. Collapsing all edges of $E$ then gives a tree $T$ as wanted.

\section{The chain condition}

We prove Theorem 1.5. In this section we only consider groups of the form $\operatorname{Out}\left(G ; \mathcal{H}^{(\mathrm{t})}\right)$, so we use the simpler notation $\operatorname{Mc}(\mathcal{H})$. Since we do not yet know that every $\operatorname{Mc}(\mathcal{H})$ is 
a McCool group, we assume that every $\mathcal{H}_{i}$ is a finite set of finitely generated subgroups (this is needed to apply Lemma 2.3).

Since $\operatorname{Mc}\left(\mathcal{H}^{\prime}\right)=\operatorname{Mc}\left(\mathcal{H} \cup \mathcal{H}^{\prime}\right)$ if $\operatorname{Mc}(\mathcal{H}) \supset \operatorname{Mc}\left(\mathcal{H}^{\prime}\right)$, we may assume $\mathcal{H}_{i} \subset \mathcal{H}_{i+1}$. We will use the following procedure several times. We associate an invariant to each family $\mathcal{H}_{i}$ and we show that, as $i$ varies, the number of distinct values of the invariant is bounded (by which we mean that there is a bound depending only on $G$ ). We then continue the proof under the additional assumption that the value of the invariant is independent of $i$.

- The first invariant is the Grushko deformation space $\mathcal{D}_{i}$ relative to $\mathcal{H}_{i}$ (see Section 2.2). The assumption $\mathcal{H}_{i} \subset \mathcal{H}_{i+1}$ implies that $\mathcal{D}_{i}$ dominates $\mathcal{D}_{i+1}$. As in the proof of Proposition 4.6, it follows from [16] that the number of times that $\mathcal{D}_{i}$ changes is bounded. We may therefore assume that $\mathcal{D}_{i}$ is constant.

Let $G_{1}, \ldots, G_{n}$ be the free factors in a Grushko decomposition $G=G_{1} * \cdots * G_{n} * F_{p}$ relative to $\mathcal{H}_{i}$ (they do not depend on $i$ up to conjugation since $\mathcal{D}_{i}$ is constant). The subgroup of $\operatorname{Mc}\left(\mathcal{H}_{i}\right)$ consisting of automorphisms sending each factor $G_{j}$ to a conjugate has bounded index and it is determined by the McCool groups $\operatorname{Mc}_{G_{j}}\left(\mathcal{H}_{i \mid G_{j}}\right)$, so we are reduced to the case when $G$ is freely indecomposable relative to $\mathcal{H}_{i}$.

- We then consider the canonical JSJ tree $T_{i}$ (over abelian subgroups) relative to $\mathcal{H}_{i}^{+a b}$, ie to $\mathcal{H}_{i}$ and all non-cyclic abelian subgroups (see $\operatorname{Section} 2.2$ ); it is $\operatorname{Mc}\left(\mathcal{H}_{i}\right)-$ invariant. We cannot use Proposition 4.8 to say that the number of distinct $T_{i}$ is bounded, because they are not RC trees, so we shall now replace $T_{i}$ by an RC JSJ tree $U_{i}$.

Any edge $e$ of $T_{i}$ joins a vertex $v_{1}$ whose stabilizer is a maximal abelian subgroup to a vertex $v_{0}$ with non-abelian stabilizer. The group $G_{e}$ is a maximal abelian subgroup of $G_{v_{0}}$, but not necessarily of $G_{v_{1}}$. Let $\bar{G}_{e}$ be the root-closure of $G_{e}$ in $G_{v_{1}}$ (hence also in $G$ ). As in [12, Section 4.3], we can fold all edges in the $\bar{G}_{e}$-orbit of $e$ together. Doing this for all edges of $T_{i}$ yields an $\mathrm{RC}$ tree $U_{i}$ which is $\operatorname{Mc}\left(\mathcal{H}_{i}\right)$-invariant.

This construction may also be described in terms of graphs of groups, as follows. We now view $e=v_{0} v_{1}$ as an edge of $T_{i} / G$. Subdivide it by adding a midpoint $u$ carrying $\bar{G}_{e}$. This creates two edges $v_{0} u$ and $u v_{1}$, carrying $G_{e}$ and $\bar{G}_{e}$, respectively. Do this for every edge $e$ of $T_{i} / G$. Collapsing all edges $u v_{1}$ yields $T_{i} / G$, whereas collapsing all edges $v_{0} u$ yields $U_{i} / G$.

The quotient graph $U_{i} / G$ is the same as $T_{i} / G$, but labels are different. Edge groups are replaced by their root-closure and non-abelian vertex groups have gotten bigger (roots have been adjoined: each fold replaces some $G_{v_{0}}$ by $G_{v_{0}} * G_{e} \bar{G}_{e}$ ). Just like $T_{i}$, the tree $U_{i}$ is equal to its tree of cylinders because folding only occurs within cylinders; in particular, $U_{i}$ is determined by its deformation space. 
Note that $U_{i}$ may have redundant vertices and is not necessarily minimal (this happens if $T_{i} / G$ has a terminal vertex carrying an abelian group, and the incident edge group has finite index). In this case we replace $U_{i}$ by its minimal subtree.

We claim that $U_{i}$ is an RC JSJ tree relative to $\mathcal{H}_{i}^{+ \text {ab }}$, in the sense of Definition 4.7. It satisfies conditions (1) and (2) since its edge stabilizers are finite extensions of edge stabilizers of $T_{i}$. Any tree satisfying these two conditions is dominated by $T_{i}$ because $T_{i}$ is a JSJ tree. But any RC tree dominated by $T_{i}$ is also dominated by $U_{i}$ (with notations as above, $e$ and $g e$ must have the same image if $g \in \bar{G}_{e}$ ).

- Proposition 4.8 lets us assume that $U_{i}$ is a fixed tree $U$. It is invariant under every $\operatorname{Mc}\left(\mathcal{H}_{i}\right)$. We let $\operatorname{Out}^{0}(U)$ be the finite-index subgroup of $\operatorname{Out}(U)$ consisting of automorphisms preserving $U$ and acting trivially on $\Gamma=U / G$. The number of edges of $\Gamma$ is uniformly bounded, by Proposition 4.5, so the index of $\operatorname{Out}^{0}(U)$ in $\operatorname{Out}(U)$ is bounded and it is enough to prove the chain condition for $\operatorname{Mc}^{0}\left(\mathcal{H}_{i}\right):=$ $\operatorname{Mc}\left(\mathcal{H}_{i}\right) \cap \operatorname{Out}^{0}(U)$.

Let $V$ be the set of vertices of $\Gamma$. As recalled in Section 2.3, there are maps $\rho_{v}: \operatorname{Out}^{0}(U) \rightarrow \operatorname{Out}\left(G_{v}\right)$ and a product map $\rho: \operatorname{Out}^{0}(U) \rightarrow \prod_{v \in V} \operatorname{Out}\left(G_{v}\right)$. Since $U$ is relative to $\mathcal{H}_{i}$, the group of twists $\mathcal{T}=\operatorname{ker} \rho$ is contained in $\operatorname{Mc}^{0}\left(\mathcal{H}_{i}\right)$.

Lemma 5.1 There exist subgroups $\operatorname{Out}^{1}\left(G_{v}\right) \subset \operatorname{Out}\left(G_{v}\right)$, independent of $i$, such that (1) $\prod_{v \in V} \operatorname{Out}^{1}\left(G_{v}\right)$ is contained in $\rho\left(\operatorname{Mc}^{0}\left(\mathcal{H}_{i}\right)\right)$ for every $i$;

(2) the index of $\operatorname{Out}^{1}\left(G_{v}\right)$ in $\rho_{v}\left(\mathrm{Mc}^{0}\left(\mathcal{H}_{i}\right)\right)$ is uniformly bounded.

This lemma implies Theorem 1.5 because $\operatorname{Mc}^{0}\left(\mathcal{H}_{i}\right)$ contains $\rho^{-1}\left(\prod_{v \in V} \operatorname{Out}^{1}\left(G_{v}\right)\right)$ with bounded index.

Proof of Lemma 5.1 Let $\mathcal{H}_{i, v}:=\left(\mathcal{H}_{i}\right)_{\| G_{v}}$ be the set of (conjugacy classes of) subgroups of $G_{v}$ which are conjugate to an element of $\mathcal{H}_{i}$ and which fix no other point in $T$ (see Section 2.1). Since two such subgroups are conjugate in $G_{v}$ if and only if they are conjugate in $G$, we may view $\mathcal{H}_{i, v}$ as a subset of $\mathcal{H}_{i}$.

Since, as explained in Section 2.3, $\rho\left(\operatorname{Mc}^{0}\left(\mathcal{H}_{i}\right)\right)$ contains $\prod_{v \in V} \operatorname{Mc}\left(\operatorname{Inc}_{v} \cup \mathcal{H}_{i, v}\right)$, it suffices to fix $v \in V$ and to construct $\operatorname{Out}^{1}\left(G_{v}\right)$ with $\operatorname{Out}^{1}\left(G_{v}\right) \subset \operatorname{Mc}\left(\operatorname{Inc}_{v} \cup \mathcal{H}_{i, v}\right)$ and the index of $\operatorname{Out}^{1}\left(G_{v}\right)$ in $\rho_{v}\left(\mathrm{Mc}^{0}\left(\mathcal{H}_{i}\right)\right)$ uniformly bounded. We distinguish several cases:

- First suppose that $G_{v} \simeq \mathbb{Z}^{k}$ is abelian, so $\operatorname{Out}\left(G_{v}\right)=\operatorname{Aut}\left(G_{v}\right)=\operatorname{GL}(k, \mathbb{Z})$. Let $A_{i}$ be the root-closure of the subgroup of $G_{v}$ generated by incident edge groups and subgroups in $\mathcal{H}_{i, v}$. It is a direct factor and increases with $i$, so we may assume that 
it is independent of $i$. We define $\operatorname{Out}^{1}\left(G_{v}\right) \subset \operatorname{Out}\left(G_{v}\right)$ as the subgroup consisting of automorphisms equal to the identity on $A_{i}$. It is equal to $\operatorname{Mc}\left(\operatorname{Inc}_{v} \cup \mathcal{H}_{i, v}\right)$ and contained in $\rho_{v}\left(\mathrm{Mc}^{0}\left(\mathcal{H}_{i}\right)\right)$. We must show that the index is bounded.

The group $A_{i}$ is invariant under $\rho\left(\mathrm{Mc}^{0}\left(\mathcal{H}_{i}\right)\right)$ and we have to bound the order of the image of $\operatorname{Mc}^{0}\left(\mathcal{H}_{i}\right)$ in $\operatorname{Out}\left(A_{i}\right)$. Any incident edge group $\bar{G}_{e}$ of $G_{v}$ contains an edge stabilizer $G_{e}$ of $T_{i}$ with finite index, and the image of the map $\rho_{e}: \operatorname{Mc}^{0}\left(\mathcal{H}_{i}\right) \rightarrow \operatorname{Out}\left(G_{e}\right)$ is finite by Lemma 2.3. Since $A_{i}$ is generated by incident edge groups and elements which are fixed by $\operatorname{Mc}^{0}\left(\mathcal{H}_{i}\right)$, this implies that the image of $\operatorname{Mc}^{0}\left(\mathcal{H}_{i}\right)$ in $\operatorname{Out}\left(A_{i}\right)$ is finite. Its cardinality is uniformly bounded because there is a bound for the order of finite subgroups of $\operatorname{GL}(k, \mathbb{Z})$, so the index of $\operatorname{Out}^{1}\left(G_{v}\right)$ in $\rho_{v}\left(\operatorname{Mc}^{0}\left(\mathcal{H}_{i}\right)\right)$ is bounded.

- We now consider a non-abelian vertex stabilizer $G_{v}$. It follows from the way $U_{i}$ was constructed that $G_{v}$ is, for each $i$, the fundamental group of a graph of groups $\Lambda_{i, v}$. This graph is a tree. It has a central vertex $v_{i}$, which may be viewed as a vertex of $T_{i} / G$ with $G_{v_{i}}$ non-abelian. All edges $e$ join $v_{i}$ to a vertex $u_{e}$ carrying a root-closed abelian group, and the index of $G_{e}$ in $G_{u_{e}}$ is finite. The graph of groups $\Lambda_{i, v}$ is invariant under the action of $\operatorname{Mc}^{0}\left(\mathcal{H}_{i}\right)$ on $G_{v}$.

We say that $G_{v}$ (or $v$ ) is rigid with sockets or $Q H$ with sockets, depending on the type of $v_{i}$ as a vertex of $T_{i}$ (since the number of vertices of $T_{i} / G$ is bounded, we may assume that this type is independent of $i$ ).

- If $G_{v}$ is rigid with sockets, we define $\operatorname{Out}^{1}\left(G_{v}\right)$ as the trivial group and we have to explain why $\rho_{v}\left(\mathrm{Mc}^{0}\left(\mathcal{H}_{i}\right)\right)$ is a finite group of bounded order. Assume first that $U=T_{i}$ (ie $U$ is also a regular JSJ tree). Lemma 2.3 then implies that $\rho_{v}\left(\operatorname{Mc}^{0}\left(\mathcal{H}_{i}\right)\right)$ is a finite subgroup of $G_{v}$, but we need to bound its order only in terms of $G$ (independently of the sequence $\mathcal{H}_{i}$ ). To get this uniform bound, we note that there are only finitely many possibilities for $G_{v}$ up to isomorphism by [24]. Moreover, $\operatorname{Out}\left(G_{v}\right)$ is virtually torsion-free by [25, Corollary 4.5], so there is a bound for the order of its finite subgroups.

In general (ie without assuming $U=T_{i}$ ), we study $\rho_{v}\left(\operatorname{Mc}^{0}\left(\mathcal{H}_{i}\right)\right)$ through its action on the graph of groups $\Lambda_{i, v}$ as in Section 2.3 (note that edges are not permuted). The group of twists is trivial because edge groups are maximal abelian in $G_{v_{i}}$ and terminal vertex groups are abelian (see [27, Proposition 3.1]), so we only have to control the action of $\mathrm{Mc}^{0}\left(\mathcal{H}_{i}\right)$ on vertex groups of $\Lambda_{i, v}$.

Applying Lemma 2.3 to the JSJ decomposition $T_{i}$, we get finiteness of the image of $\operatorname{Mc}^{0}\left(\mathcal{H}_{i}\right)$ in $\operatorname{Out}\left(G_{v_{i}}\right)$ and in $\operatorname{Out}\left(G_{e}\right)$ for every edge $e$ of $T_{i}$, and hence of $\Lambda_{i, v}$. The action of an automorphism on the edge groups of $\Lambda_{i, v}$ determines the action on the abelian vertex groups because they contain the incident edge group with finite index. This proves that $\rho_{v}\left(\mathrm{Mc}^{0}\left(\mathcal{H}_{i}\right)\right)$ is finite, and boundedness follows as above. 
- There remains the case when $G_{v}$ is $\mathrm{QH}$ with sockets. The group $G_{v_{i}}$ is then isomorphic to the fundamental group of a compact surface $\Sigma_{i}$ and incident edge groups are boundary subgroups. The topology of $\Sigma_{i}$ may vary with $i$, but the number of boundary components of $\Sigma_{i}$ is bounded (by a simple accessibility argument, or because the rank of $G_{v_{i}}$ as a free group is bounded, by [24]).

If $J$ is a subgroup of $G$, denote by $\mathcal{U}_{i}(J)$ the set of elements of $J$ that are $\mathcal{H}_{i}^{+ \text {ab }}$ universally elliptic (ie elliptic in every $G$-tree with abelian edge stabilizers which is relative to $\mathcal{H}_{i}$ and to non-cyclic abelian subgroups). We view it as a union of $J$-conjugacy classes. Since $\mathcal{H}_{i} \subset \mathcal{H}_{i+1}$, we have $\mathcal{U}_{i}(J) \subset \mathcal{U}_{i+1}(J)$. We shall show that the sequence $\mathcal{U}_{i}\left(G_{v}\right)$ stabilizes.

We first study $\mathcal{U}_{i}\left(G_{v_{i}}\right)$ : we claim that $\mathcal{U}_{i}\left(G_{v_{i}}\right)$ is the union of the conjugacy classes of boundary subgroups of $G_{v_{i}}=\pi_{1}\left(\Sigma_{i}\right)$. Indeed, any boundary subgroup is an incident edge group of $v_{i}$ (up to conjugacy) or has a finite-index subgroup conjugate to a group in $\mathcal{H}_{i}$ (otherwise, $G$ would be freely decomposable relative to $\mathcal{H}_{i}$; see [21, Proposition 7.5]). It follows that $\mathcal{U}_{i}\left(G_{v_{i}}\right)$ contains all boundary subgroups (incident edge groups are $\mathcal{H}_{i}^{+\mathrm{ab}}$-universally elliptic because $T_{i}$ is a JSJ tree relative to $\mathcal{H}_{i}^{+\mathrm{ab}}$ ). Conversely, by [21, Proposition 7.6], any $g \in \mathcal{U}_{i}\left(G_{v_{i}}\right)$ is contained in a boundary subgroup of $\pi_{1}\left(\Sigma_{i}\right)$. This proves our claim and shows, in particular, that $\mathcal{U}_{i}\left(G_{v_{i}}\right)$ is the union of a bounded number of conjugacy classes of maximal cyclic subgroups $L_{j}(i)$ of $G_{v_{i}}$.

We now consider $\mathcal{U}_{i}\left(G_{v}\right)$. The $\mathcal{H}_{i}^{+ \text {ab }}$-universally elliptic elements of $G_{v}$ are contained (up to conjugacy) in $G_{v_{i}}$ or in one of the terminal vertex groups of $\Lambda_{i, v}$, so $\mathcal{U}_{i}\left(G_{v}\right)$ is the union of the conjugates of the root-closures (in $G_{v}$ ) of the groups $L_{j}(i)$. Since $\mathcal{H}_{i} \subset \mathcal{H}_{i+1}$, we have $\mathcal{U}_{i}\left(G_{v}\right) \subset \mathcal{U}_{i+1}\left(G_{v}\right)$. As $\mathcal{U}_{i}\left(G_{v}\right)$ is the union of the conjugates of a bounded number of cyclic subgroups, we may assume that $\mathcal{U}_{i}\left(G_{v}\right)=\mathcal{U}\left(G_{v}\right)$ does not depend on $i$.

Elements of $\rho_{v}\left(\mathrm{Mc}^{0}\left(\mathcal{H}_{i}\right)\right)$ send each cyclic group in $\mathcal{U}\left(G_{v}\right)$ to a conjugate (conjugacy classes are not permuted because the action on $T_{i} / G$ is trivial). They act trivially on groups in $\mathcal{H}_{i, v}$, but they may map an element $g$ belonging to a terminal vertex group of $\Lambda_{v, i}$ to $g^{-1}$ (geometrically, they correspond to homeomorphisms of $\Sigma_{i}$ which may reverse orientation on boundary components).

We define $\operatorname{Out}^{1}\left(G_{v}\right) \subset \operatorname{Out}\left(G_{v}\right)$ as the group of automorphisms acting trivially on each cyclic group in $\mathcal{U}\left(G_{v}\right)$ (geometrically, we restrict to homeomorphisms of $\Sigma_{i}$ equal to the identity on the boundary). It is contained in $\operatorname{Mc}\left(\operatorname{Inc}_{v} \cup \mathcal{H}_{i, v}\right)$, because $\mathcal{U}_{i}\left(G_{v}\right)$ contains the incident edge groups of $G_{v}$ in $U$, hence contained in $\rho_{v}\left(\operatorname{Mc}^{0}\left(\mathcal{H}_{i}\right)\right)$, and the index is bounded in terms of the number of conjugacy classes of cyclic subgroups in $\mathcal{U}\left(G_{v}\right)$. 
Remark 5.2 Groups of the form $\operatorname{Out}(G ; \mathcal{H})$, with $\mathcal{H}$ a finite family of abelian groups, do not satisfy the descending chain condition: consider $G=\mathbb{Z}^{2}=\langle x, y\rangle$ and $\mathcal{H}_{i}=\left\{\left\langle x, y^{2^{i}}\right\rangle\right\}$.

\section{Proof of the other results}

We first note the following consequence of the chain condition:

Proposition 6.1 If $\mathcal{C}$ is an infinite family of conjugacy classes, there exists a finite subfamily $\mathcal{C}^{\prime} \subset \mathcal{C}$ such that $\operatorname{Mc}(\mathcal{C})=\operatorname{Mc}\left(\mathcal{C}^{\prime}\right)$.

Recall that $\operatorname{Mc}(\mathcal{C})$ is the group of outer automorphisms fixing all conjugacy classes belonging to $\mathcal{C}$.

Proof Write $\mathcal{C}$ as an increasing union of finite families $\mathcal{C}_{i}$ and note that $\operatorname{Mc}(\mathcal{C})$ is the intersection of the descending chain $\operatorname{Mc}\left(\mathcal{C}_{i}\right)$.

To prove Corollary 1.6, saying in particular that every $\mathrm{McCool}$ group is an elementary McCool group, we need the following fact:

Lemma 6.2 Let $G$ be a toral relatively hyperbolic group. Let $H$ be a subgroup and $\alpha \in \operatorname{Aut}(G)$. If $\alpha(h)$ and $h$ are conjugate in $G$ for every $h \in H$, then $\alpha$ acts on $H$ as conjugation by some $g \in G$.

Proof We may assume that there is a non-trivial $h \in H$ such that $\alpha(h)=h$. If $H$ is abelian, malnormality of maximal abelian subgroups implies that $\alpha$ is the identity on $H$. If not, the result follows from [31, Lemma 5.2] (which is valid for any homomorphism $\varphi: H \rightarrow G$, not just automorphisms of $H$ ); see also [2, Corollary 7.4].

Corollary 1.6 Let $G$ be a toral relatively hyperbolic group. If $\mathcal{H}$ is any family of subgroups of $G$, there exists a finite set of conjugacy classes such that $\operatorname{Mc}(\mathcal{H})=\operatorname{Mc}(\mathcal{C})$.

Recall that $\operatorname{Mc}(\mathcal{H})$ is also denoted by $\operatorname{Out}\left(G ; \mathcal{H}^{(\mathrm{t})}\right)$. We favor the notation $\operatorname{Mc}(\mathcal{H})$ in this subsection.

Proof Given an arbitrary family $\mathcal{H}$, let $\mathcal{C}_{\mathcal{H}}$ be the set of all conjugacy classes having a representative belonging to some $H_{i}$. By Lemma 6.2, $\operatorname{Mc}(\mathcal{H})=\operatorname{Mc}\left(\mathcal{C}_{\mathcal{H}}\right)$. We apply Proposition 6.1 to get $\operatorname{Mc}(\mathcal{H})=\operatorname{Mc}(\mathcal{C})$ with $\mathcal{C}$ finite.

Together with Theorem 3.11, this implies our most general finiteness result. 
Corollary 6.3 Let $G$ be a toral relatively hyperbolic group. Let $\mathcal{H}$ be an arbitrary collection of subgroups of $G$. Let $\mathcal{K}$ be a finite collection of abelian subgroups of $G$. Let $T$ be a simplicial tree on which $G$ acts with abelian edge stabilizers, with each group in $\mathcal{H} \cup \mathcal{K}$ fixing a point.

Then the group $\operatorname{Out}\left(T, \mathcal{H}^{(\mathrm{t})}, \mathcal{K}\right)=\operatorname{Out}(T) \cap \operatorname{Out}\left(G ; \mathcal{H}^{(\mathrm{t})}, \mathcal{K}\right)$ of automorphisms leaving $T$ invariant, acting trivially on each group of $\mathcal{H}$ and sending each $K \in \mathcal{K}$ to a conjugate (in an arbitrary way) is of type VF.

Proof By Corollary 1.6, we may write $\operatorname{Out}\left(G ; \mathcal{H}^{(\mathrm{t})}\right)=\operatorname{Mc}(\mathcal{C})$ for some finite family of conjugacy classes $\left[c_{i}\right]$, with each $c_{i}$ belonging to a group of $\mathcal{H}$ and hence elliptic in $T$. Defining $\mathcal{L}=\left\{\left\langle c_{i}\right\rangle\right\}$, we see that $\operatorname{Mc}(\mathcal{C})$ is a finite-index $\operatorname{subgroup}$ of $\operatorname{Out}(G ; \mathcal{L})$, so $\operatorname{Out}\left(T, \mathcal{H}^{(\mathrm{t})}, \mathcal{K}\right)$ is a finite-index subgroup of $\operatorname{Out}(T, \mathcal{K} \cup \mathcal{L})$. By Theorem 3.11, this group has type VF and therefore so does $\operatorname{Out}\left(T, \mathcal{H}^{(\mathrm{t})}, \mathcal{K}\right)$.

Proposition 1.7 and Theorem 1.8 will be proved at the end of the section.

Proposition 1.10 Given a toral relatively hyperbolic group $G$, there exists a number $C$ such that, if a subgroup $\widehat{M} \subset \operatorname{Out}(G)$ contains a group $\operatorname{Mc}(\mathcal{H})$ with finite index, then the index $[\widehat{M}: \operatorname{Mc}(\mathcal{H})]$ is bounded by $C$.

Proof By Corollary 1.6, we may write $\operatorname{Mc}(\mathcal{H})=\operatorname{Mc}\left(\mathcal{C}^{\prime}\right)$ for some finite set $\mathcal{C}^{\prime}$. Let $\mathcal{C}$ be the orbit of $\mathcal{C}^{\prime}$ under $\widehat{M}$. Since $\operatorname{Mc}\left(\mathcal{C}^{\prime}\right)$ fixes $\mathcal{C}^{\prime}$, this is a finite $\widehat{M}$-invariant collection of conjugacy classes. We thus have

$$
\operatorname{Mc}(\mathcal{C}) \subset \operatorname{Mc}\left(\mathcal{C}^{\prime}\right) \subset \widehat{M} \subset \widehat{\operatorname{Mc}}(\mathcal{C})
$$

and it suffices to bound the index $[\widehat{\operatorname{Mc}}(\mathcal{C}): \operatorname{Mc}(\mathcal{C})]$.

As in the beginning of Section 5, let $G=G_{1} * \cdots * G_{n} * F_{r}$ be a Grushko decomposition of $G$ relative to $\mathcal{C}$ and let $\mathcal{G}=\left\{G_{1}, \ldots, G_{n}\right\}$. The group $\widehat{\operatorname{Mc}}(\mathcal{C})$ permutes the conjugacy classes of the groups in $\mathcal{G}$. Since the cardinality of $\mathcal{G}$ is bounded and $G$ has finitely many free factors up to isomorphism, we may assume that $G$ is one-ended relative to $\mathcal{C}$.

We now consider the JSJ decomposition $T_{\text {can }}$ over abelian groups relative to $\mathcal{C}$ and non-cyclic abelian groups. It is invariant under $\widehat{\operatorname{Mc}}(\mathcal{C})$, so we may study $\widehat{\operatorname{Mc}}(\mathcal{C})$ through its action on $T_{\text {can }}$ (see Section 2.3).

The number of edges of $\Gamma_{\text {can }}=T_{\text {can }} / G$ being bounded by the first case of Proposition 4.5, we may replace $\widehat{\operatorname{Mc}}(\mathcal{C})$ and $\operatorname{Mc}(\mathcal{C})$ by their subgroups $\widehat{M c}^{0}(\mathcal{C})$ and $\operatorname{Mc}^{0}(\mathcal{C})$ acting trivially on $\Gamma$. The group of twists $\mathcal{T}$ is contained in $\operatorname{Mc}^{0}(\mathcal{C})$, so as in the proof of 
Lemma 5.1 it suffices to construct $\operatorname{Out}^{1}\left(G_{v}\right) \subset \operatorname{Mc}_{G_{v}}\left(\operatorname{Inc}_{v} \cup \mathcal{C}_{\| G_{v}}\right)$ with the index of $\operatorname{Out}^{1}\left(G_{v}\right)$ in $\rho_{v}\left(\widehat{M}^{0}(\mathcal{C})\right)$ uniformly bounded. We distinguish the same cases as in the proof of Lemma 5.1.

If $G_{v}$ is abelian, isomorphic to $\mathbb{Z}^{k}$ with $k \geq 2$, let $H<G_{v}$ be the set of elements whose orbit under $\rho_{v}\left(\widehat{\mathrm{Mc}}^{0}(\mathcal{C})\right)$ is finite. This is a subgroup of $G_{v}$, isomorphic to some $\mathbb{Z}^{p}$, which is invariant under $\rho_{v}\left(\widehat{\mathrm{Mc}}^{0}(\mathcal{C})\right)$ and contains the incident edge groups by Lemma 2.3. We define $\operatorname{Out}^{1}\left(G_{v}\right)=\operatorname{Mc}_{G_{v}}(\{H\})$. It is contained in $\operatorname{Mc}_{G_{v}}\left(\operatorname{Inc}_{v} \cup \mathcal{C}_{\| G_{v}}\right)$. The image of $\rho_{v}\left(\widehat{\operatorname{Mc}}^{0}(\mathcal{C})\right)$ in $\operatorname{Aut}(H)=\operatorname{GL}(p, \mathbb{Z})$ is finite, and its order bounds the index of $\operatorname{Out}^{1}\left(G_{v}\right)$ in $\rho_{v}\left(\widehat{\mathrm{Mc}}^{0}(\mathcal{C})\right)$. This concludes the proof in this case, since there is a bound for the order of finite subgroups of $\operatorname{GL}(p, \mathbb{Z})$.

If $G_{v}$ is rigid, we let $\operatorname{Out}^{1}\left(G_{v}\right)$ be trivial. The image of $\widehat{M c}^{0}(\mathcal{C})$ in $\operatorname{Out}\left(G_{v}\right)$ is finite by Lemma 2.3, and bounded by [24] as in the proof of Lemma 5.1.

If $G_{v}=\pi_{1}(\Sigma)$ is $\mathrm{QH}$, we define $\operatorname{Out}^{1}\left(G_{v}\right)=\mathcal{P} \mathcal{M}^{+}(\Sigma)=\operatorname{Mc}_{G_{v}}\left(\operatorname{Inc}_{v} \cup \mathcal{C}_{\| G_{v}}\right)$. Elements of $\rho_{v}\left(\widehat{\mathrm{Mc}}^{0}(\mathcal{C})\right)$ may reverse orientation, or permute boundary components of $\Sigma$.

Corollary 6.4 Extended elementary $\mathrm{McCool}$ groups $\widehat{\mathrm{Mc}}(\mathcal{C})$ of $G$ satisfy a uniform chain condition.

Proof Given a descending chain $\widehat{\operatorname{Mc}}\left(\mathcal{C}_{i}\right)$, define $\mathcal{C}_{i}^{\prime}=\mathcal{C}_{0} \cup \cdots \cup \mathcal{C}_{i}$ and note that

$$
\operatorname{Mc}\left(\mathcal{C}_{i}^{\prime}\right)=\bigcap_{j \leq i} \operatorname{Mc}\left(\mathcal{C}_{j}\right) \subset \widehat{\operatorname{Mc}}\left(\mathcal{C}_{i}\right)=\bigcap_{j \leq i} \widehat{\operatorname{Mc}}\left(\mathcal{C}_{j}\right) \subset \widehat{\operatorname{Mc}}\left(\mathcal{C}_{i}^{\prime}\right)
$$

The corollary follows from Theorem 1.5, since by Proposition 1.10 the index of $\operatorname{Mc}\left(\mathcal{C}_{i}^{\prime}\right)$ in $\widehat{M} c\left(\mathcal{C}_{i}^{\prime}\right)$ is bounded.

We now prove Corollary 1.11, stating that, for any $A<\operatorname{Out}(G)$, there is a subgroup $A_{0}<A$ of bounded finite index such that, for the action of $A_{0}$ on the set of conjugacy classes of $G$, every orbit is a singleton or is infinite.

Proof of Corollary 1.11 Let $\mathcal{C}_{A}$ be the (possibly infinite) set of conjugacy classes of $G$ whose $A$-orbit is finite. Partition $\mathcal{C}_{A}$ into $A$-orbits and let $\mathcal{C}_{p}$ be the union of the first $p$ orbits. The image of $A$ in the group of permutations of $\mathcal{C}_{p}$ is contained in that of $\widehat{\operatorname{Mc}}\left(\mathcal{C}_{p}\right)$, so by Proposition 1.10 its order is bounded by some fixed $C$. This $C$ also bounds the order of the image of $A$ in the group of permutations of $\mathcal{C}_{A}$. 
Recall that $\operatorname{Ac}\left(\mathcal{H}, H_{0}\right) \subset \operatorname{Aut}(G)$ is the group of automorphisms acting trivially on $\mathcal{H}$ (in the sense of Definition 1.2, ie by conjugation) and fixing the elements of $H_{0}$. Proposition 1.13 states that, if $G$ is non-abelian, then $\operatorname{Ac}\left(\mathcal{H}, H_{0}\right)$ is an extension

$$
1 \longrightarrow K \longrightarrow \operatorname{Ac}\left(\mathcal{H}, H_{0}\right) \longrightarrow \operatorname{Mc}\left(\mathcal{H}^{\prime}\right) \longrightarrow 1
$$

with $\operatorname{Mc}\left(\mathcal{H}^{\prime}\right) \subset \operatorname{Out}(G)$ a McCool group and $K$ the centralizer of $H_{0}$. Corollary 1.14 states that the groups $\operatorname{Ac}\left(\mathcal{H}, H_{0}\right)$ are of type VF and satisfy a uniform chain condition.

Proof of Proposition 1.13 Let $\mathcal{H}^{\prime}=\mathcal{H} \cup\left\{H_{0}\right\}$. Map $\operatorname{Ac}\left(\mathcal{H}, H_{0}\right) \subset \operatorname{Aut}(G)$ to $\operatorname{Out}(G)$. The image is $\operatorname{Mc}\left(\mathcal{H}^{\prime}\right)$. The kernel $K$ is the set of inner automorphisms equal to the identity on $H_{0}$. Since $G$ has trivial center, it is isomorphic to the centralizer of $H_{0}$.

Proof of Corollary 1.14 The group $\operatorname{Mc}\left(\mathcal{H}^{\prime}\right)$ has type VF by Theorem 1.3. The group $K$ is abelian or equal to $G$, so has type F because $G$ does [10]. Proposition 1.13 and Corollary 3.2 imply that $\operatorname{Ac}\left(\mathcal{H}, H_{0}\right)$ has type VF. Moreover, a chain of centralizers has length at most 2 since the centralizer of $H_{0}$ is trivial, $G$ or a maximal abelian subgroup. The uniform chain condition for McCool groups (Theorem 1.5) then implies the uniform chain condition for groups of the form $\operatorname{Ac}\left(\mathcal{H}, H_{0}\right)$.

We now deduce the bounded chain condition for fixed subgroups.

Proof of Theorem 1.8 Let $J_{0} \varsubsetneqq J_{1} \varsubsetneqq \cdots \varsubsetneqq J_{p}$ be a strictly ascending chain of fixed subgroups. Let $\operatorname{Ac}\left(\varnothing, J_{i}\right)$ be the subgroup of $\operatorname{Aut}(G)$ consisting of automorphisms equal to the identity on $J_{i}$. Since $J_{i}$ is a fixed subgroup, $\operatorname{Ac}\left(\varnothing, J_{i}\right) \supsetneq \operatorname{Ac}\left(\varnothing, J_{i+1}\right)$. Corollary 1.14 then gives a bound on the length of the chain.

Remark One can adapt the arguments of Section 5 to prove Theorem 1.8 directly (without passing through McCool groups).

We now prove Proposition 1.7, saying that $\operatorname{Out}\left(F_{n}\right)$ contains infinitely many nonisomorphic McCool groups for $n \geq 4$ and infinitely many non-conjugate McCool groups for $n \geq 3$.

Proof of Proposition 1.7 Let $H$ be the free group on three generators $a, b, c$. Given a non-trivial element $w \in\langle a, b\rangle$, let $P_{w}$ be the cyclic HNN extension $P_{w}=$ $\left\langle a, b, c, t \mid t c t^{-1}=w\right\rangle$. It is free of rank 3, with basis $a, b, t$. Let $\varphi_{w}$ be the automorphism of $P_{w}$ fixing $a$ and $b$ and mapping $t$ to $w t$ (it equals the identity on $H$ since it fixes $\left.c=t^{-1} w t\right)$. The image $\Phi_{w}$ of $\varphi_{w}$ in $\operatorname{Out}\left(P_{w}\right)$ preserves the Bass-Serre tree $T$ of the HNN extension (it belongs to its group of twists $\mathcal{T}$ ). 
We apply this construction with $w=a^{k} b^{k}$ for $k$ a positive integer. As $k$ varies, the cyclic subgroups $\left\langle\Phi_{w}\right\rangle$ are pairwise non-conjugate in $\operatorname{Out}\left(P_{w}\right) \simeq \operatorname{Out}\left(F_{3}\right)$, as seen by considering the action on the abelianization.

We shall now prove the second assertion of the proposition for $n=3$, by showing that $\left\langle\Phi_{w}\right\rangle$ is a McCool group of $P_{w}$, namely $\left\langle\Phi_{w}\right\rangle=\operatorname{Mc}_{P_{w}}(\{H\}) \subset \operatorname{Out}\left(F_{3}\right)$. The extension to $n>3$ is straightforward, by adding generators to $H$.

Consider splittings of $P_{w}$ over abelian (ie cyclic) subgroups relative to $H$. The tree $T$ is a JSJ tree because its vertex stabilizers are universally elliptic [21, Lemma 4.7]; in particular, $P_{w}$ is freely indecomposable relative to $H$. Moreover, $T$ equals its tree of cylinders (up to adding redundant vertices) because $w$ is not a proper power, so $T$ is the canonical JSJ tree $T_{\text {can }}$. The McCool group $\operatorname{Mc}_{P_{w}}(\{H\})$ therefore leaves $T$ invariant and it is easily checked using [27] that $\operatorname{Mc}_{P_{w}}(\{H\})=\mathcal{T}=\left\langle\Phi_{w}\right\rangle$.

To prove the first assertion of the proposition, consider $R_{w}=P_{w} *\langle d\rangle \simeq F_{4}$, the family $\mathcal{H}=\{H,\langle d\rangle\}$ and the $\mathrm{McCool}$ group $\operatorname{Mc}_{R_{w}}(\mathcal{H}) \subset \operatorname{Out}\left(F_{4}\right)$. The decomposition $R_{w}=P_{w} *\langle d\rangle$ is a Grushko decomposition of $R_{w}$ relative to $\mathcal{H}$ because $P_{w}$ is freely indecomposable relative to $H$. This decomposition is invariant under $\operatorname{Mc}_{R_{w}}(\mathcal{H})$ because it is a one-edge splitting (see [14, Corollary 1.3]).

The stabilizer $\operatorname{Out}(T)$ of the Bass-Serre tree $T$ in $\operatorname{Out}\left(R_{w}\right)$ is naturally isomorphic to

$$
\operatorname{Aut}\left(P_{w}\right) \times \operatorname{Aut}(\langle d\rangle) \simeq \operatorname{Aut}\left(P_{w}\right) \times \mathbb{Z} / 2 \mathbb{Z}
$$

(see [27]); the natural map $\operatorname{Out}(T) \rightarrow \operatorname{Out}\left(P_{w}\right)$ kills the factor $\mathbb{Z} / 2 \mathbb{Z}$ and coincides with the quotient map $\operatorname{Aut}\left(P_{w}\right) \rightarrow \operatorname{Out}\left(P_{w}\right)$ on the other factor. The McCool group $\operatorname{Mc}_{R_{w}}(\mathcal{H})$ is isomorphic to the preimage of $\operatorname{Mc}_{P_{w}}(\{H\})=\left\langle\Phi_{w}\right\rangle$ in $\operatorname{Aut}\left(P_{w}\right)$, hence to the mapping torus

$$
Q_{w}=\left\langle a, b, t, u \mid u a=a u, u b=b u, u t u^{-1}=a^{k} b^{k} t\right\rangle .
$$

The abelianization of $Q_{w}$ is $\mathbb{Z}^{3} \times \mathbb{Z} / k \mathbb{Z}$, so the isomorphism type of $Q_{w}$ changes when $k$ varies. This proves the first assertion of the proposition for $n=4$. The extension to larger $n$ is again straightforward.

\section{Appendix: Groups with finitely many McCool groups}

In this appendix we describe cases when $\operatorname{Out}(G)$ only contains finitely many McCool subgroups. In particular, we show that the values of $n$ given in Proposition 1.7 are optimal. 
Proposition A.1 If $G$ is a torsion-free, one-ended hyperbolic group, then $\operatorname{Out}(G)$ only contains finitely many McCool groups up to conjugacy.

Proposition A.2 Out $\left(F_{2}\right)$ only contains finitely many McCool groups up to conjugacy.

Proposition A.3 $\operatorname{Out}\left(F_{3}\right)$ only contains finitely many McCool groups up to isomorphism.

The proof of Proposition A.1 requires the fact that $\operatorname{Out}(G)$, and, more generally, extended $\mathrm{McCool}$ groups $\widehat{\operatorname{Mc}}(\mathcal{C})$, only contain finitely many conjugacy classes of finite subgroups. This will appear in [17].

Proof of Proposition A.1 We assume that $\operatorname{Out}(G)$ contains infinitely many nonconjugate elementary $\mathrm{McCool}$ groups $\operatorname{Mc}\left(\mathcal{C}_{i}\right)$ and we derive a contradiction (this implies the proposition, by Corollary 1.6).

It is proved in [33, Corollary 4.9] that there are only finitely many minimal actions of $G$ on trees with cyclic edge stabilizers, up to the action of $\operatorname{Out}(G)$, so we may assume that the canonical cyclic JSJ tree relative to $\mathcal{C}_{i}$ (the tree $T_{\text {can }}$ of Section 2.2) is a given tree $T$. This tree is invariant under all groups $\operatorname{Mc}\left(\mathcal{C}_{i}\right)$, so $\operatorname{Mc}\left(\mathcal{C}_{i}\right) \subset \operatorname{Out}(T)$. In this proof, we cannot restrict to $\operatorname{Out}^{0}(T)$.

Given a vertex $v$ of $T$, we define $\mathcal{C}_{i, v}$ as the restriction $\mathcal{C}_{i \mid G_{v}}$ if $G_{v}$ is cyclic and as $\mathcal{C}_{i \| G_{v}}$ if $G_{v}$ is not cyclic (recall from Section 2.1 that conjugacy classes represented by elements fixing an edge of $T$ do not belong to $\mathcal{C}_{i \| G_{v}}$ ). The tree being bipartite, $\mathcal{C}_{i}$ is the disjoint union of the $\mathcal{C}_{i, v}$.

We say that $v$ is used if $\mathcal{C}_{i, v}$ is non-empty. Since there are finitely many $G$-orbits of vertices, we may assume that usedness is independent of $i$; we let $V_{u}$ be a set of representatives of orbits of used vertices. We may also assume that the type of vertices with non-cyclic stabilizer (rigid or $\mathrm{QH}$ ) is independent of $i(\mathrm{QH}$ vertices with $\Sigma$ a pair of pants are rigid; we do not consider them as $\mathrm{QH}$ ).

We claim that $\mathrm{QH}$ vertices $G_{v}$ of $T$ are not used. Indeed, any boundary subgroup of $G_{v}$ is an incident edge stabilizer of $T$ : otherwise, $G_{v}$ would split as a free product relative to $\operatorname{Inc}_{v}$, contradicting one-endedness of $G$. Elements in $\mathcal{C}_{i}$ are universally elliptic (relative to $\mathcal{C}_{i}$ ) and the only universally elliptic subgroups of $G_{v}$ are contained in boundary subgroups of $G_{v}$ because $G_{v}$ is flexible (see [21, Proposition 7.6]), so $\mathcal{C}_{i \| G_{v}}$ is empty. 
For $v \in V_{u}$, define $\operatorname{Out}_{i}\left(G_{v}\right) \subset \operatorname{Out}\left(G_{v}\right)$ as the set of automorphisms which fix each conjugacy class in $\mathcal{C}_{i, v}$ and leave the set of incident edge stabilizers globally invariant. Any automorphism in $\operatorname{Mc}\left(\mathcal{C}_{i}\right)$ is an automorphism of $T$ which leaves $G_{v}$ invariant (up to conjugacy) and induces an automorphism belonging to $\operatorname{Out}_{i}\left(G_{v}\right)$. Conversely, any automorphism of $T$ satisfying these properties for every $v \in V_{u}$ lies in $\operatorname{Mc}\left(\mathcal{C}_{i}\right)$. This means that $\operatorname{Mc}\left(\mathcal{C}_{i}\right)$ is completely determined by the knowledge of the groups $\operatorname{Out}_{i}\left(G_{v}\right)$ for $v \in V_{u}$.

We complete the proof by showing that there are only finitely many possibilities for each $\operatorname{Out}_{i}\left(G_{v}\right)$. This is clear if $G_{v}$ is cyclic, and QH vertices are not used, so there remains to consider the case where $G_{v}$ is rigid.

In this case, $\operatorname{Out}_{i}\left(G_{v}\right)$ is finite by Lemma 2.3 (otherwise $G_{v}$ would have a cyclic splitting relative to $\operatorname{Inc}_{v}$ and $\mathcal{C}_{i, v}$, contradicting rigidity). Since $G_{v}$ is hyperbolic, $\operatorname{Out}\left(G_{v}\right)$ has finitely many conjugacy classes of finite subgroups [17]. We deduce that there are finitely many possibilities for $\operatorname{Out}_{i}\left(G_{v}\right)$, up to conjugacy in $\operatorname{Out}\left(G_{v}\right)$. Unfortunately, this is not enough to get finiteness for $\operatorname{Mc}\left(\mathcal{C}_{i}\right)$ up to conjugacy in $\operatorname{Out}(G)$, because the conjugator may fail to extend to an automorphism of $G$.

To remedy this, we consider $\operatorname{Mc}\left(\operatorname{Inc}_{v}\right)$ and $\widehat{\operatorname{Mc}}\left(\operatorname{Inc}_{v}\right)$, with $\operatorname{Inc}_{v}$ the family of incident edge groups as in Section 2.1 and $\widehat{\operatorname{Mc}}\left(\operatorname{Inc} v_{v}\right)=\widehat{\operatorname{Out}}\left(G_{v} ; \operatorname{Inc}_{v}\right)$ the set of outer automorphisms of $G_{v}$ preserving $\operatorname{Inc}_{v}$ (see Definition 2.1; edge groups may be permuted and the generator of an edge group may be mapped to its inverse).

The group $\operatorname{Out}_{i}\left(G_{v}\right) \subset \operatorname{Out}\left(G_{v}\right)$ is finite and contained in $\widehat{\operatorname{Mc}}\left(\operatorname{Inc}_{v}\right)$ (but not necessarily in $\left.\mathrm{Mc}\left(\operatorname{Inc}_{v}\right)\right)$. By [17], $\widehat{\operatorname{Mc}}\left(\operatorname{Inc}_{v}\right)$ has only finitely many conjugacy classes of finite subgroups. It follows that there are only finitely many possibilities for $\operatorname{Out}_{i}\left(G_{v}\right)$ up to conjugation by an element of $\widehat{\mathrm{M}}\left(\operatorname{Inc}_{v}\right)$, hence also up to conjugation by an element of $\operatorname{Mc}\left(\operatorname{Inc}_{v}\right)$ since $\operatorname{Mc}\left(\operatorname{Inc}_{v}\right)$ has finite index in $\widehat{\operatorname{Mc}}\left(\operatorname{Inc}_{v}\right)$.

We may therefore assume that $\operatorname{Out}_{i}\left(G_{v}\right)$ is independent of $i$ if $G_{v}$ is cyclic and $v \in V_{u}$, and that all groups $\operatorname{Out}_{i}\left(G_{v}\right)$ are conjugate by elements of $\operatorname{Mc}\left(\operatorname{Inc}_{v}\right)$ if $v \in V_{u}$ is rigid. Any element of $\operatorname{Mc}\left(\operatorname{Inc}_{v}\right)$ extends "by the identity" to an automorphism of $G$ which leaves $T$ invariant and acts trivially (as conjugation by an element of $G$ ) on $G_{w}$ if $w$ is not in the orbit of $v$. Since $\operatorname{Mc}\left(\mathcal{C}_{i}\right)$ is determined by the groups $\operatorname{Out}_{i}\left(G_{v}\right)$ for $v \in V_{u}$, we conclude that all groups $\operatorname{Mc}\left(\mathcal{C}_{i}\right)$ are conjugate in $\operatorname{Out}(G)$.

Proof of Proposition A.2 We view $\operatorname{Out}\left(F_{2}\right) \simeq \mathrm{GL}(2, \mathbb{Z})$ as the mapping class group of a punctured torus $\Sigma$ (with orientation-reversing maps allowed). Let $c$ be a peripheral conjugacy class (representing the commutator of basis elements of $F_{2}$ ).

We consider a $\operatorname{McCool} \operatorname{group} \operatorname{Mc}(\mathcal{H}) \subset \operatorname{Out}\left(F_{2}\right)$. We may assume that $\operatorname{Mc}(\mathcal{H})$ is infinite. By the classification of elements of $\operatorname{GL}(2, \mathbb{Z})$ or by the Bestvina-Paulin 
method and Rips theory, $F_{2}$ then splits over a cyclic group relative to $\mathcal{H}$ and $c$ (see for instance [25, Theorem 3.9]). Such a splitting is dual to a non-peripheral simple closed curve $\gamma \subset \Sigma$.

If there are two different splittings, they are dual to curves $\gamma$ and $\gamma^{\prime}$ whose union fills $\Sigma$, so $\mathcal{H}$ only contains peripheral subgroups. It follows that $\operatorname{Mc}(\mathcal{H})$ is either $\operatorname{Out}\left(F_{2}\right) \simeq \operatorname{GL}(2, \mathbb{Z})$ or $\operatorname{SL}(2, \mathbb{Z})$. If the splitting is unique, $\operatorname{Mc}(\mathcal{H})$ fixes $\gamma$ (viewed as an unoriented curve up to isotopy). Since the splitting dual to $\gamma$ is relative to $\mathcal{H}$, the Dehn twist $T_{\gamma}$ around $\gamma$ is contained in $\operatorname{Mc}(\mathcal{H})$. The stabilizer $\operatorname{Stab}(\gamma)$ of $\gamma$ in the mapping class group contains $\left\langle T_{\gamma}\right\rangle$ with finite index (the index is 4 because a homeomorphism may reverse the orientation of $\Sigma$ and/or of $\gamma$ ). We thus have $\left\langle T_{\gamma}\right\rangle \subset \operatorname{Mc}(\mathcal{H}) \subset \operatorname{Stab}(\gamma)$, with both indices finite. Finiteness of $\operatorname{Mc}(\mathcal{H})$ up to conjugacy follows, since $\gamma$ is unique up to the action of the mapping class group.

The remainder of this appendix is devoted to the proof of Proposition A.3. We first record a few useful facts.

Lemma A.4 Fix $n$. Up to isomorphism, $\operatorname{Out}\left(F_{n}\right)$ only contains finitely many virtually solvable subgroups.

Proof Virtually solvable subgroups are virtually abelian [1; 5]. More precisely, they contain $\mathbb{Z}^{k}$ with $k \leq 2 n-3$ as a subgroup of bounded index (see [5, Proof of Theorem 1.1, page 94]). This implies finiteness, for instance by [32, Theorem 8.6].

Lemma A.5 Let $A$ be virtually cyclic and $B$ be virtually $F_{n}$ for some $n$. Up to isomorphism, there are only finitely many groups which are extensions of $A$ by $B$.

Proof This follows from standard extension theory [8, Sections III.10 and IV.6], noting that $\operatorname{Out}(A)$ is finite and $B$ has a finite-index subgroup with trivial $H^{2}$.

Proof of Proposition A.3 Now consider a $\operatorname{McCool} \operatorname{group} \operatorname{Mc}(\mathcal{H}) \subset \operatorname{Out}\left(F_{3}\right)$. The first step is to reduce to the case where $F_{3}$ is freely indecomposable relative to $\mathcal{H}$. If this does not hold, let $\Gamma$ be a Grushko decomposition relative to $\mathcal{H}$ (see Section 2.2). It is not unique; we choose one with as few edges as possible.

If all vertex groups are cyclic, groups in $\mathcal{H}$ are generated (up to conjugacy) by powers of elements belonging to some fixed basis of $F_{3}$, and finiteness holds. Otherwise, there is a vertex group $G_{v} \simeq F_{2}$. Our choice of $\Gamma$ implies that $\Gamma$ has a single edge (it is an HNN extension, or an amalgam $F_{2} * \mathbb{Z}$ with a finite-index subgroup of $\mathbb{Z}$ belonging to $\mathcal{H})$. It follows that $\Gamma$ is $\operatorname{Mc}(\mathcal{H})$-invariant $[14 ; 28]$ and $\operatorname{Mc}(\mathcal{H})$ is determined by its image in $\operatorname{Out}\left(F_{2}\right)$. This image is the $\mathrm{McCool}$ group $\operatorname{Mc}\left(\mathcal{H}_{\mid F_{2}}\right)$, so finiteness follows from Proposition A.2. 
We continue the proof under the assumption that $F_{3}$ is freely indecomposable relative to $\mathcal{H}$. Let $\Gamma_{\text {can }}$ be the canonical $\operatorname{Mc}(\mathcal{H})$-invariant cyclic JSJ decomposition relative to $\mathcal{H}$ (see Section 2.2). Vertex groups $G_{v}$ are cyclic, rigid or $\mathrm{QH}$.

One easily checks the formula $\sum_{v}\left(\operatorname{rk} G_{v}-1\right)=2$. In particular, $\operatorname{rk} G_{v} \leq 3$ for all $v$ and, if some $G_{v}$ is isomorphic to $F_{3}$, then all other vertex groups are cyclic.

If $G_{v} \simeq \pi_{1}(\Sigma)$ is a $\mathrm{QH}$ vertex group, it is isomorphic to $F_{2}$ or $F_{3}$, so there are 9 possibilities for the compact surface $\Sigma$ :

(1) Pair of pants.

(2) Sphere with 4 boundary components.

(3) Projective plane with 2 boundary components.

(4) Projective plane with 3 boundary components.

(5) Torus with 1 boundary component.

(6) Torus with 2 boundary components.

(7) Klein bottle with 1 boundary component.

(8) Klein bottle with 2 boundary components.

(9) Non-orientable surface of genus 3 with 1 boundary component.

Each incident edge group $G_{e}$ is (up to conjugacy) a boundary subgroup of $\pi_{1}(\Sigma)$. Conversely, there are two possibilities for a boundary subgroup $C$. If it is an incident edge group, it equals $G_{e}$ for a unique incident edge. If not, we say that the corresponding boundary component of $\Sigma$ is free; in this case, some finite-index subgroup of $C$ belongs to $\mathcal{H}$.

As in Section 2.3, the finite-index subgroup $\operatorname{Mc}^{0}(\mathcal{H})$ of $\operatorname{Mc}(\mathcal{H})$ acting trivially on $\Gamma_{\text {can }}$ maps to $\prod_{v} \operatorname{Out}\left(G_{v}\right)$ with kernel the group of twists $\mathcal{T}$. The image $\operatorname{in} \operatorname{Out}\left(G_{v}\right)$ is finite if $G_{v}$ is cyclic or rigid, and virtually the mapping class group of $\Sigma$ if $G_{v}$ is QH, and $\mathcal{T}$ is isomorphic to some $\mathbb{Z}^{k}$ (see [25, Section 4.3]).

By mapping class group, we mean the group of isotopy classes of homeomorphisms of a compact surface $\Sigma$ mapping each boundary component to itself in an orientationpreserving way. We denote it by $\mathcal{P M}^{+}(\Sigma)$ as in Section 2.2.

By Lemma A.4, we may assume that there is a $\mathrm{QH}$ vertex $v$ with $\mathcal{P M}^{+}(\Sigma)$ nonsolvable. As explained above, there are 9 possibilities for $\Sigma$. Cases 1,3 and 7 are ruled out because $\mathcal{P} \mathcal{M}^{+}(\Sigma)$ is virtually cyclic (see [34], or argue as in the proof of Proposition A.2, noting that a finite-index subgroup of $\mathcal{P} \mathcal{M}^{+}(\Sigma)$ fixes a conjugacy class of $F_{2}$ which is not a power of the commutator). 
If $\Gamma_{\text {can }}$ is trivial (ie if the QH subgroup $G_{v}$ is the whole group), $\operatorname{Mc}(\mathcal{H})$ is the mapping class group of $\Sigma$. We therefore assume that $\Gamma_{\text {can }}$ is non-trivial.

Lemma A.6 If $G_{v}$ has rank 3, then $\Sigma$ has a free boundary component.

Proof This follows from [4, Lemma 4.1], a generalization of the standard fact that a cyclic amalgam $A *\langle c\rangle$ of free groups is free only if $c$ belongs to a basis in $A$ or $B$.

This lemma rules out case 9 .

Now suppose that all vertices of $\Gamma_{\text {can }}$ other than $v$ are terminal vertices carrying $\mathbb{Z}$ (by Lemma A.6, this holds in cases 6 and 8). In this case the group of twists $\mathcal{T}$ is trivial (see [27, Proposition 3.1]). The group $\operatorname{Mc}(\mathcal{H})$ contains $\mathcal{P M}^{+}(\Sigma)$ with finite index and there are finitely many possibilities: they depend on whether edges of $\Gamma_{\text {can }}$ may be permuted and whether elements in edge groups may be mapped to their inverse.

We must now deal with cases 2,4 and 5 . We start with 4 . The only possibility left is that $\Gamma_{\text {can }}$ has two vertices $v$ and $w$ joined by 2 edges, with $G_{w}$ cyclic. Every automorphism leaving $\Gamma_{\text {can }}$ invariant maps $G_{v}$ to itself (up to conjugacy), and we consider the natural map from $\operatorname{Mc}(\mathcal{H})$ to $\operatorname{Out}\left(G_{v}\right)$. As above, the image contains $\mathcal{P} \mathcal{M}^{+}(\Sigma)$ with finite index and there are finitely many possibilities. The kernel is the group of twists $\mathcal{T}$, which is isomorphic to $\mathbb{Z}$. Since $\mathcal{P} \mathcal{M}^{+}(\Sigma)$ is isomorphic to $F_{3}$ by [34, Theorem 7.5], we conclude by Lemma A.5.

The argument in case 2 is similar. Besides $v$ and $w$, there may be another vertex $w^{\prime}$, with $G_{w^{\prime}}$ cyclic and a single edge between $v$ and $w^{\prime}$. The group $\mathcal{P} \mathcal{M}^{+}(\Sigma)$ is again free; it is isomorphic to $F_{2}$ (see for instance [13, Section 4.2.4]).

In case 5 (a once-punctured torus), there is a single edge incident to $v$. Collapsing all other edges yields a $\operatorname{Mc}(\mathcal{H})$-invariant decomposition as an amalgam $F_{3}=G_{v} *\langle a\rangle G_{w}$ with $G_{w} \simeq F_{2}$. By the standard fact recalled above, $a$ belongs to a basis of $G_{w}$ (and is equal to a commutator in $G_{v}$ ). The group $\operatorname{Mc}(\mathcal{H})$ acts trivially on the graph underlying this amalgam and the map $\rho$ (see $\operatorname{Section} 2.3)$ maps $\operatorname{Mc}(\mathcal{H})$ to $\operatorname{Out}\left(G_{v}\right) \times \operatorname{Out}\left(G_{w}\right)$, with kernel the group of twists $\mathcal{T}$, isomorphic to $\mathbb{Z}$. The image in $\operatorname{Out}\left(G_{v}\right)$ is isomorphic to $\operatorname{GL}(2, \mathbb{Z})$ or $\operatorname{SL}(2, \mathbb{Z})$.

We now consider the image $L$ of $\operatorname{Mc}(\mathcal{H})$ in $\operatorname{Out}\left(G_{w}\right)$. It preserves the conjugacy class of $\langle a\rangle$. If $L$ is finite (necessarily of order at most 6 ), then $\operatorname{Mc}(\mathcal{H})$ maps onto $\operatorname{GL}(2, \mathbb{Z})$ or $\operatorname{SL}(2, \mathbb{Z})$ with virtually cyclic kernel $K$; there are finitely many possibilities for $K$ up to isomorphism (it maps to $L$ with cyclic kernel), and we conclude by Lemma A.5. As explained in the proof of Proposition A.2, if $L$ is infinite, it is virtually cyclic, contains 
a "Dehn twist" $T_{a}$ and has index at most 4 in the stabilizer of the conjugacy class of $\langle a\rangle$ in $\operatorname{Out}\left(G_{w}\right)$. Since $\operatorname{Mc}(\mathcal{H})$ is determined by its image in $\operatorname{Out}\left(G_{v}\right) \times \operatorname{Out}\left(G_{w}\right)$ and this image contains $\operatorname{SL}(2, \mathbb{Z}) \times\left\langle T_{a}\right\rangle$, this leaves only finitely many possibilities.

\section{References}

[1] E Alibegović, Translation lengths in $\operatorname{Out}\left(F_{n}\right)$, Geom. Dedicata 92 (2002) 87-93 MR1934012

[2] Y Antolin, A Minasyan, A Sisto, Commensurating endomorphisms of acylindrically hyperbolic groups and applications, preprint (2013) arXiv:1310.8605

[3] M Bestvina, M Feighn, Bounding the complexity of simplicial group actions on trees, Invent. Math. 103 (1991) 449-469 MR1091614

[4] M Bestvina, M Feighn, Outer limits, preprint (1992) Available at http:// andromeda.rutgers.edu/ feighn/papers/outer.pdf

[5] M Bestvina, M Feighn, M Handel, Solvable subgroups of $\operatorname{Out}\left(F_{n}\right)$ are virtually abelian, Geom. Dedicata 104 (2004) 71-96 MR2043955

[6] A Borel, Density and maximality of arithmetic subgroups, J. Reine Angew. Math. 224 (1966) 78-89 MR0205999

[7] A Borel, J-P Serre, Corners and arithmetic groups, Comment. Math. Helv. 48 (1973) 436-491 MR0387495

[8] K S Brown, Cohomology of groups, Graduate Texts in Mathematics 87, Springer, New York (1982) MR672956

[9] M Culler, K Vogtmann, Moduli of graphs and automorphisms of free groups, Invent. Math. 84 (1986) 91-119 MR830040

[10] F Dahmani, Classifying spaces and boundaries for relatively hyperbolic groups, Proc. London Math. Soc. 86 (2003) 666-684 MR1974394

[11] F Dahmani, D Groves, The isomorphism problem for toral relatively hyperbolic groups, Publ. Math. Inst. Hautes Études Sci. (2008) 211-290 MR2434694

[12] F Dahmani, V Guirardel, The isomorphism problem for all hyperbolic groups, Geom. Funct. Anal. 21 (2011) 223-300 MR2795509

[13] B Farb, D Margalit, A primer on mapping class groups, Princeton Mathematical Series 49, Princeton Univ. Press (2012) MR2850125

[14] M Forester, Deformation and rigidity of simplicial group actions on trees, Geom. Topol. 6 (2002) 219-267 MR1914569

[15] R Geoghegan, Topological methods in group theory, Graduate Texts in Mathematics 243, Springer, New York (2008) MR2365352 
[16] V Guirardel, Actions of finitely generated groups on $\mathbb{R}$-trees, Ann. Inst. Fourier (Grenoble) 58 (2008) 159-211 MR2401220

[17] V Guirardel, G Levitt, Extension finiteness for relatively hyperbolic groups, in preparation

[18] V Guirardel, G Levitt, Strata and canonical subtrees of $\mathbb{R}$-trees, in preparation

[19] V Guirardel, G Levitt, Deformation spaces of trees, Groups Geom. Dyn. 1 (2007) 135-181 MR2319455

[20] V Guirardel, G Levitt, The outer space of a free product, Proc. Lond. Math. Soc. 94 (2007) 695-714 MR2325317

[21] V Guirardel, G Levitt, JSJ decompositions: definitions, existence, uniqueness, I: The JSJ deformation space, preprint (2009) arXiv:0911.3173

[22] V Guirardel, G Levitt, JSJ decompositions: definitions, existence, uniqueness, II: Compatibility and acylindricity, preprint (2010) arXiv:1002.4564

[23] V Guirardel, G Levitt, Trees of cylinders and canonical splittings, Geom. Topol. 15 (2011) 977-1012 MR2821568

[24] V Guirardel, G Levitt, Vertex finiteness for splittings of relatively hyperbolic groups, preprint (2013) arXiv:1311.2835 to appear in Israel J. Math.

[25] V Guirardel, G Levitt, Splittings and automorphisms of relatively hyperbolic groups, Groups Geom. Dyn. 9 (2015) 599-663 MR3356977

[26] M Handel, L Mosher, Subgroup decomposition in $\operatorname{Out}\left(F_{n}\right)$ : introduction and research announcement, preprint (2013) arXiv:1302.2681

[27] G Levitt, Automorphisms of hyperbolic groups and graphs of groups, Geom. Dedicata 114 (2005) 49-70 MR2174093

[28] G Levitt, Characterizing rigid simplicial actions on trees, from: "Geometric methods in group theory", (J Burillo, S Cleary, M Elder, J Taback, E Ventura, editors), Contemp. Math. 372, Amer. Math. Soc. (2005) 27-33 MR2139674

[29] A Martino, E Ventura, Fixed subgroups are compressed in free groups, Comm. Algebra 32 (2004) 3921-3935 MR2097438

[30] J McCool, Some finitely presented subgroups of the automorphism group of a free group, J. Algebra 35 (1975) 205-213 MR0396764

[31] A Minasyan, D Osin, Normal automorphisms of relatively hyperbolic groups, Trans. Amer. Math. Soc. 362 (2010) 6079-6103 MR2661509

[32] D Segal, Polycyclic groups, Cambridge Tracts in Mathematics 82, Cambridge Univ. Press (1983) MR713786

[33] Z Sela, Acylindrical accessibility for groups, Invent. Math. 129 (1997) 527-565 MR1465334 
[34] B Szepietowski, A presentation for the mapping class group of a non-orientable surface from the action on the complex of curves, Osaka J. Math. 45 (2008) 283-326 MR2441942

Institut de Recherche Mathématique de Rennes, Université de Rennes 1 et CNRS (UMR 6625) 263 avenue du Général Leclerc, CS 74205, 35042 Rennes Cedex, France Laboratoire de Mathématiques Nicolas Oresme, Université de Caen et CNRS (UMR 6139) BP 5186, 14032 Caen Cedex 5, France vincent.guirardel@univ-rennes1.fr, levitt@unicaen.fr

Received: 15 October 2014 